TRANSACTIONS OF THE

AMERICAN MATHEMATICAL SOCIETY

Volume 362, Number 12, December 2010, Pages 6397-6424

S 0002-9947(2010)05213-4

Article electronically published on July 14, 2010

\title{
STABILITY OF NONCHARACTERISTIC BOUNDARY LAYERS IN THE STANDING-SHOCK LIMIT
}

\author{
KEVIN ZUMBRUN
}

\begin{abstract}
We investigate one- and multi-dimensional stability of noncharacteristic boundary layers in the limit approaching a standing planar shock wave $\bar{U}\left(x_{1}\right), x_{1}>0$, obtaining necessary conditions of (i) weak stability of the limiting shock, (ii) weak stability of the constant layer $u \equiv U_{-}:=\lim _{z \rightarrow-\infty} \bar{U}(z)$, and (iii) nonnegativity of a modified Lopatinski determinant similar to that of the inviscid shock case. For Lax 1-shocks, we obtain equally simple sufficient conditions; for $p$-shocks, $p>1$, the situation appears to be more complicated. Using these results, we determine the stability of certain gas dynamical boundary layers, generalizing earlier work of Serre-Zumbrun and CostanzinoHumphreys-Nguyen-Zumbrun.
\end{abstract}

\section{CONTEnTs}

1. Introduction

1.1. Equations and assumptions $\quad 6400$

1.2. The Evans condition 6401

1.3. Main results 6403

1.4. Discusssion and open problems 6407

2. Construction of the Evans function 6408

2.1. Expression as a first-order system 6408

2.2. Conjugation to constant coefficients 6408

2.3. Definition of the Evans function 6409

2.4. Behavior near zero $\quad 6409$

3. Basic convergence result $\quad 6410$

4. Behavior near the origin $\quad 6411$

5. Application to gas dynamics $\quad 6416$

5.1. Viscous shock profiles

5.2. One-dimensional stability $\quad 6419$

$\begin{array}{ll}\text { The case } \phi \geq 1 & 6420\end{array}$

The case $\phi \leq 1 \quad 6420$

Common gases and the kinetic approximation $\quad 6421$

Received by the editors September 15, 2008.

2010 Mathematics Subject Classification. Primary 35Q35; Secondary 35B35.

The author's research was partially supported under NSF grants number DMS-0070765 and DMS-0300487.

(C)2010 American Mathematical Society Reverts to public domain 28 years from publication 
Conclusions 6421

5.3. Multi-dimensional stability 6421

Appendix A. Computation of $\ell_{+}$in one dimension 6422

References 6423

\section{INTRODUCTION}

Consider a boundary layer, or stationary solution,

$$
\tilde{U}=\bar{U}\left(x_{1}\right), \quad \lim _{z \rightarrow+\infty} \bar{U}(z)=U_{+}, \quad \bar{U}(0)=U_{0}
$$

of a hyperbolic-parabolic system of conservation laws on the quarter-space

$$
\tilde{U}_{t}+\sum_{j} F_{j}(\tilde{U})_{x_{j}}=\sum_{j k}\left(B_{j k}(\tilde{U}) \tilde{U}_{x_{k}}\right)_{x_{j}}, \quad x \in \mathbb{R}_{+}^{d}=\left\{x_{1}>0\right\}, \quad t>0,
$$

$\tilde{U}, F_{j} \in \mathbb{R}^{n}, B_{j k} \in \mathbb{R}^{n \times n}$,

$$
\tilde{U}=\left(\begin{array}{c}
\tilde{u} \\
\tilde{v}
\end{array}\right), \quad B=\left(\begin{array}{cc}
0 & 0 \\
b_{j k}^{1} & b_{j k}^{2}
\end{array}\right), \quad \tilde{u} \in \mathbb{R}^{n-r}, \tilde{v} \in \mathbb{R}^{r},
$$

with initial data $\tilde{U}(x, 0)=\tilde{U}_{0}(x)$ and boundary conditions as specified in (1.6) below, that is, noncharacteristic both in the hyperbolic sense

$$
\operatorname{det} d F^{1}\left(\bar{U}_{+}\right) \neq 0
$$

and with respect to the original (partially) parabolic problem as described in (H1)(H3) below. Such layers occur, for instance, in gas- and magnetohydrodynamics with inflow or outflow boundary conditions, for example in flow around an airfoil with micro-suction or blowing; see [GMWZ5, YZ, NZ1, NZ2 for further discussion.

As for any gas-dynamical flow, an important question is stability of these solutions under perturbation of the initial or boundary data. This question has been investigated in GR, MZ1, GMWZ5, GMWZ6, YZ, NZ1, NZ2 for arbitraryamplitude boundary layers using Evans function techniques, with the result that under quite general circumstances (see model assumptions below) linearized and nonlinear stability reduce to a generalized spectral stability condition phrased in terms of the Evans function, a Wronskian associated with the family of eigenvalue ODE obtained by Fourier transform in the transverse directions $\tilde{x}:=\left(x_{2}, \ldots, x_{d}\right)$. See also the small-amplitude results of [GG, R3, MN, KNZ, KK obtained by energy methods.

The Evans function is readily evaluable numerically; see, e.g., CHNZ, HLyZ1, HLyZ2. As pointed out in [SZ, CHNZ, GMWZ5, it is also evaluable analytically in certain interesting asymptotic limits. For example, it was shown in GMWZ5 that the Evans function converges in the small-amplitude limit as $\bar{U}$ approaches the constant layer $U \equiv U_{+}$to the Evans function of the constant layer, uniformly on compact sets of frequencies $\Re \lambda \geq 0$, and, as a consequence, stability of smallamplitude layers is determined by stability of the limiting constant layer. This result was used in turn to show that noncharacteristic boundary layers of general symmetric-dissipative systems (defined below) are spectrally stable in the smallamplitude limit. 
A different asymptotic limit considered for special cases in [SZ, CHNZ] is the standing shock limit $X \rightarrow+\infty$ in the case

$$
\bar{U}^{X}(x)=\hat{U}\left(x_{1}-X\right), \quad \lim _{z \rightarrow \pm \infty} \hat{U}(z)=U_{ \pm}
$$

that $\bar{U}$ is the restriction to $x_{1}>0$ of a standing shock solution $\hat{U}(\cdot-X)$. It is natural to guess that there might be some relation between boundary-layer stability in this limit and stability of the limiting shock wave, and indeed it was shown in CHNZ for the case of isentropic ideal gas dynamics that the boundary-layer Evans function, suitably normalized, converges in the standing-shock limit to the Evans function of the limiting shock wave on compact subsets of frequencies $\Re \lambda \geq 0$, in complete analogy with the small-amplitude limit. On the other hand, it was shown in SZ] for the case of full (nonisentropic) ideal gas dynamics that boundary layers can in some parameter regimes be unstable in the standing-shock limit despite stability (see HLyZ1) of the limiting shock wave.

In the present paper, we revisit the standing-shock limit in the general case, obtaining a result subsuming and illuminating these previous ones. Moreover, we carry out our investigations in multi-dimensions, whereas the analyses of [SZ, CHNZ] were specific to the one-dimensional case. Specifically, we show that the boundary-layer Evans function, suitably normalized, converges in the standing-shock limit, uniformly on compact subsets of frequencies $\eta \in \mathbb{R}^{d-1}, \Re \lambda \geq 0$, to the product of the Evans function of the limiting shock wave and the Evans function of the constant layer $U \equiv U_{-}$at the left endstate of the shock. For symmetric-dissipative systems, this implies by the stability of constant layers that the Evans function can be further renormalized so as to converge simply to the Evans function of the limiting shock, similarly as was shown in [CHNZ] in the special one-dimensional isentropic ideal gas case.

A consequence is that stability of both the limiting shock and the constant layer $U \equiv U_{-}$are necessary conditions for stability of boundary layers in the standingshock limit. On the other hand, these are not sufficient, as even stable shock waves have an (one-dimensional) eigenvalue at $\eta=0, \lambda=0$ due to translationinvariance, whereas stable boundary layers do not. A further necessary condition, therefore, is nonnegativity of the stability index (defined below) counting parity of the number of (one-dimensional) unstable roots $\eta=0, \Re \lambda>0$, indicating that the zero eigenvalue of the limiting shock does not perturb into the positive halfplane. Negativity of the stability index in the standing-shock limit, indicating an odd number of unstable eigenvalues, was what was shown in [SZ in order to obtain one-dimensional instability.

Here, we develop these ideas substantially further, determining for Lax 1-shocks a simple and general stability determinant $\hat{\Delta}(\tilde{\xi}, \lambda, \eta)$ extending the Lopatinski determinant $\Delta(\tilde{\xi}, \lambda)$ of the inviscid shock case, $\tilde{\xi} \in \mathbb{R}, \eta \in \mathbb{R}, \lambda \in \mathbb{C}$, with $\Re \lambda, \eta \geq 0$, for which nonvanishing on the strictly positive half-space $\Re \lambda, \eta>0$ is necessary and nonvanishing on the nonnegative half-space $\Re \lambda, \eta \geq 0$ together with stability of the limiting shock $\hat{U}$ and the constant layer $U \equiv U_{-}$is sufficient for stability in the standing-shock limit, in one- and multi-dimensions. We then use this condition to investigate stability in various interesting situations. For $p$-shocks, $p>1$, the situation is considerably more tricky, involving a complicated double limit. 
1.1. Equations and assumptions. Consider a family $\bar{U}^{X}(x)$ of boundary layers (1.3) of (1.2) consisting of translations of a standing shock solution $\hat{U}$. Following GMWZ5, GMWZ6, we assume that the conservation law (1.2) can be rewritten in nonconservative form, after an invertible change of variables $\tilde{U} \rightarrow \tilde{W}$, as a quasilinear hyperbolic-parabolic system

$$
\tilde{A}_{0}(\tilde{W}) u_{t}+\sum_{j=1}^{d} \tilde{A}_{j}(\tilde{W}) \partial_{j}(\tilde{W})-\varepsilon \sum_{j, k=1}^{d} \partial_{j}\left(\tilde{B}_{j k}(\tilde{W}) \partial_{k} \tilde{W}\right)=0,
$$

$\tilde{A}_{0}$ invertible, with block structure

$$
\tilde{A}_{0}(\tilde{W})=\left(\begin{array}{cc}
\tilde{A}_{0}^{11} & 0 \\
\tilde{A}_{0}^{21} & \tilde{A}_{0}^{22}
\end{array}\right), \quad \tilde{B}_{j k}(\tilde{W})=\left(\begin{array}{cc}
0 & 0 \\
0 & \tilde{B}_{j k}^{22}
\end{array}\right),
$$

a corresponding splitting $\tilde{W}=\left(\tilde{w}^{1}, \tilde{w}^{2}\right) \in \mathbb{R}^{n-r} \times \mathbb{R}^{r}$, and decoupled boundary conditions

$$
\left\{\begin{array}{l}
\Upsilon_{1}\left(\tilde{w}^{1}\right)_{\mid x \in \partial \Omega}=g_{1}(t, x) \\
\Upsilon_{2}\left(\tilde{w}^{2}\right)_{\mid x \in \partial \Omega}=g_{2}(t, x) \\
\Upsilon_{3}\left(\tilde{w}, \partial_{x_{1}} \tilde{w}^{2}, \partial_{\tilde{x}} \tilde{w}^{2}\right)_{\mid x \in \partial \Omega}=0
\end{array} .\right.
$$

where $\Upsilon_{3}\left(\tilde{w}, \partial_{x_{1}} \tilde{w}^{2}, \partial_{\tilde{x}} \tilde{w}^{2}\right)=K_{1} \partial_{x_{1}} \tilde{w}^{2}+\sum_{j=2}^{d} K_{j}(\tilde{w}) D_{x_{j}} \tilde{w}^{2}, K_{1} \equiv$ constant, $\operatorname{dim} \Upsilon_{1}$ $=n-r$ in the inflow case and 0 in the outflow case (defined in (H2) just below), and

$$
\operatorname{dim} \Upsilon_{2}+\operatorname{dim} \Upsilon_{3}=r
$$

We make the following technical hypotheses following [Z1, Z3, GMWZ5].

(H0) $F^{j}, B^{j k}, \tilde{A}^{0}, \tilde{A}^{j}, \tilde{B}^{j k}, \tilde{W}(\cdot), \tilde{g}(\cdot, \cdot) \in C^{s}, s \geq 2$.

(H1) The eigenvalues of $\sum_{j}\left(\tilde{A}_{0}^{11}\right)^{-1} \tilde{A}_{j}^{11} \xi_{j}$ are real and semisimple for all $\xi \neq 0$ in $\mathbb{R}^{d}$.

(H2) The eigenvalues of $\left(\tilde{A}_{0}^{11}\right)^{-1} \tilde{A}_{1}^{11}$ are either strictly positive or strictly negative, that is, either $\sigma\left(\tilde{A}_{0}^{11}\right)^{-1} \tilde{A}_{1}^{11} \geq \theta_{1}>0$ (inflow case) or $\sigma\left(\tilde{A}_{0}^{11}\right)^{-1} \tilde{A}_{1}^{11} \leq-\theta_{1}<0$ (outflow case).

(H3) $\Re \sigma \sum_{j k} b_{2}^{j k} \xi_{j} \xi_{k} \geq \theta|\xi|^{2}>0$ for all $\xi \in \mathbb{R}^{n} \backslash\{0\}$.

(H4) The eigenvalues of $\sum_{j} d F_{ \pm}^{j} \xi_{j}$ are real, semisimple, and have constant multiplicity with respect to $\xi \in \mathbb{R}^{d}, \xi \neq 0$.

(H5) The eigenvalues of $d F^{1}\left(U_{ \pm}\right)$are nonzero.

(H6) $\Re \sigma\left(\sum_{j} i \xi_{j} d F^{j}\left(U_{ \pm}\right)-\sum_{j, k} \xi_{j} \xi_{k} B^{j k}\left(U_{ \pm}\right)\right) \leq-\theta \frac{|\xi|^{2}}{1+|\xi|^{2}}$ for all $\xi \in \mathbb{R}^{d}$, some $\theta>0$.

Definition 1.1. The system (1.2), (1.4) is symmetric dissipative at $U_{ \pm}$if in a neighborhood of $U_{ \pm}$there exists a real matrix $S(\tilde{U})$ depending smoothly on $\tilde{U}$ such that for all $\xi \in \mathbb{R}^{d} \backslash\{0\}, S(\tilde{U}) \tilde{A}_{0}(\tilde{U})$ is symmetric positive definite and blockdiagonal, $S(\tilde{U}) \sum_{j} \tilde{A}_{j}(\tilde{U}) \xi_{j}$ is symmetric, and $\Re S(\tilde{U}) \sum \tilde{B}_{j k}(\tilde{U}) \xi_{j} \xi_{k}$ is nonnegative with kernel of dimension $n-r$.

Alternative Hypothesis $\mathbf{H} 4^{\prime}$ For systems that are symmetric dissipative at $U_{ \pm}$, we may relax (H4) to:

(H4 $4^{\prime}$ ) About each $\xi \in \mathbb{R}^{d} \backslash\{0\}$, the eigenvalues of $\sum_{j} d F_{ \pm}^{j} \xi_{j}$ (necessarily real and semisimple, by symmetrizability) are either of constant multiplicity or else are totally nonglancing in the sense of [GMWZ6, Definition 4.3. 
Definition 1.2. The system (1.2), (1.4) is symmetric hyperbolic-parabolic if there exists a real matrix $S(\tilde{U})$ depending smoothly on $\tilde{U}$ such that for all $\xi \in \mathbb{R}^{d} \backslash\{0\}$, the matrix $S(\tilde{U}) \tilde{A}_{0}(\tilde{U})$ is symmetric positive definite and block-diagonal, $\left(S(\tilde{U}) \sum_{j} \tilde{A}_{j}(\tilde{U}) \xi_{j}\right)^{11}$ is symmetric, and the symmetric matrix $\Re S(\tilde{U}) \sum \tilde{B}_{j k}(\tilde{U}) \xi_{j} \xi_{k}$ is nonnegative with kernel of dimension $n-r$.

Examples 1.3. Hypotheses (H1)-(H6) are satisfied for standing shocks of the compressible Navier-Stokes equations with van der Waal equation of state, yielding boundary layers for which the normal velocity of the fluid is nonvanishing at $U_{0}$. This corresponds to the situation of a porous boundary through which fluid is pumped in or out, in contrast to the characteristic, no-flux boundary conditions encountered at a solid material interface for which normal velocity is set to zero. See [YZ, GMWZ5, NZ1, NZ2] for further discussion of this situation and applications to aerodynamics.

Hypotheses (H1)-(H6) with (H4) replaced by $\left(\mathrm{H} 4^{\prime}\right)$ are satisfied for extreme (i.e., 1- or $n$-family) standing Lax shocks of the viscous MHD equations with van der Waal equation of state, with similar velocity restrictions on the plasma at $U_{0}$, but fail for intermediate shocks. Hypotheses $(\mathrm{H} 1)-(\mathrm{H} 6)$ are generically satisfied for viscous MHD in dimension one, but fail always for viscous MHD in dimensions greater than or equal to two; see [MZ2, GMWZ5, GMWZ6 for further discussion. Both gas dynamics and MHD equations with van der Waals equation of state are symmetric hyperbolic-parabolic systems that are symmetric dissipative at $U_{ \pm}$for standing shocks connecting thermodynamically stable endstates [Z3, GMWZ4].

Finally, regarding the standing shock $\hat{U}$, ordering the eigenvalues of $d F\left(U_{ \pm}\right)$as

$$
a_{1}^{ \pm}<a_{2}^{ \pm}<\cdots<a_{n}^{ \pm},
$$

we assume:

(H7) Profile $\hat{U}$ is a transversal viscous Lax $p$-shock, i.e.,

$$
a_{p-1}^{-}<0<a_{p}^{-}, \quad a_{p}^{+}<0<a_{p+1}^{+}
$$

and $\hat{U}$ is a transversal connection of the standing wave ODE with boundary conditions $\tilde{U}_{ \pm}$(see [MaZ3] for a detailed discussion of the standing wave ODE).

The eigenvalues $a_{j}^{ \pm}$correspond to characteristic speeds at $U_{ \pm}$of the associated one-dimensional inviscid system $U_{t}+F_{1}(U)_{x_{1}}=0$, with $a_{p}$ the principal characteristic speed associated with the shock.

1.2. The Evans condition. The linearized eigenvalue equations of (1.2), (1.6) about $\bar{U}$ are

$$
\lambda U=L U:=\sum_{j, k}\left(B_{j k} U_{x_{k}}\right)_{x_{j}}-\sum_{j}\left(A_{j} U\right)_{x_{j}},
$$

with homogeneous boundary conditions

$$
\Upsilon^{\prime}\left(W, \partial_{\tilde{x}} w^{2}, \partial_{x_{1}} w^{2}\right)_{\mid x_{1}=0}=0
$$

expressed in linearized $\tilde{W}$-coordinates $W:=(\partial \tilde{W} / \partial \tilde{U})(\bar{U}) U$, where $W$ and $U$ denote perturbations of $\tilde{W}$ and $\tilde{U}$. 
Taking the Fourier transform in $\tilde{x}:=\left(x_{2}, \ldots, x_{d}\right)$, we obtain a family of eigenvalue ODEs

$$
\begin{aligned}
\lambda U=L_{\tilde{\xi}} U:=\overbrace{\left(B_{11} U^{\prime}\right)^{\prime}-\left(A_{1} U\right)^{\prime}}^{L_{0} U}-i \sum_{j \neq 1} A_{j} \xi_{j} U+i \sum_{j \neq 1} B_{j 1} \xi_{j} U^{\prime} \\
+i \sum_{k \neq 1}\left(B_{1 k} \xi_{k} U\right)^{\prime}-\sum_{j, k \neq 1} B_{j k} \xi_{j} \xi_{k} U
\end{aligned}
$$

with boundary conditions

$$
\Upsilon^{\prime}\left(W, i \tilde{\xi} w^{2}, \partial_{x_{1}} w^{2}\right)_{\mid x_{1}=0}=0 .
$$

1.2.1. The boundary-layer Evans function. A necessary condition for linearized stability is weak spectral stability, defined as nonexistence of unstable spectra $\Re \lambda>0$ of the linearized operator $L$ about the wave. As described in Section 2 this is equivalent to nonvanishing for all $\tilde{\xi} \in \mathbb{R}^{d-1}, \Re \lambda>0$ of the Evans function

$$
D(\tilde{\xi}, \lambda),
$$

a Wronskian associated with (1.10) with columns consisting of bases of the subspace of solutions decaying as $x_{1} \rightarrow+\infty$ and the subspace of solutions satisfying the boundary condition (1.11). Under our hypotheses, the Evans function may be defined to be $C^{\infty}$ away from the origin on $\Re \lambda \geq 0$ and analytic in $\lambda$ with continous limits (typically depending on direction) at $(0,0)$ along rays through the origin; see Se2, SZ, GMWZ5, GMWZ6].

Definition 1.4. We define strong spectral, or uniform Evans stability as

$$
|D(\tilde{\xi}, \lambda)| \geq \theta(C)>0
$$

for $(\tilde{\xi}, \lambda)$ on bounded subsets $C \subset\left\{\tilde{\xi} \in \mathbb{R}^{d-1}, \Re \lambda \geq 0\right\} \backslash\{0\}$.

Remark 1.5. Under assumptions (H0)-(H6), uniform Evans stability implies linearized and nonlinear stability in both the long time and small viscosity limits of general noncharacteristic boundary layers (not necessarily associated with standing shocks) of symmetric hyperbolic-parabolic systems that are symmetric-dissipative at $U_{+}$with Dirichlet boundary conditions $\operatorname{dim} \Upsilon_{3}=0$; see GMWZ5, GMWZ6, NZ1, NZ2, N2, For more general systems and boundary conditions, (D) augmented with a rescaled high-frequency condition has been shown in GMWZ5, GMWZ6 to imply stability in the small viscosity limit.

1.2.2. The shock Evans function. Likewise, a necessary condition for linearized stability of the shock wave $\hat{U}$ is weak spectral stability, defined as nonexistence of unstable spectra $\Re \lambda>0$ of the linearized operator $L$ about the wave, or nonvanishing for all $\tilde{\xi} \in \mathbb{R}^{d-1}, \Re \lambda>0$ of the shock Evans function

$$
\mathcal{D}(\tilde{\xi}, \lambda),
$$

a Wronskian associated with (1.10) with columns consisting of bases of the subspace of solutions decaying as $x_{1} \rightarrow+\infty$ and the subspace of solutions decaying as $x_{1} \rightarrow$ $-\infty$. Under our hypotheses, the shock Evans function may be defined to be $C^{\infty}$ and analytic in $\lambda$ away from the origin on $\Re \lambda \geq 0$ and $C^{0}$ at the origin, with first directional derivatives (typically depending on direction) at $(0,0)$ along rays through the origin; see [GZ, Z3, GMWZ4, GMWZ6]. 
Definition 1.6. Uniform Evans stability of a standing shock is defined as

$$
|\mathcal{D}(\tilde{\xi}, \lambda)| \geq \theta(C)>0
$$

for $(\tilde{\xi}, \lambda)$ on bounded subsets $C \subset\left\{\tilde{\xi} \in \mathbb{R}^{d-1}, \Re \lambda \geq 0\right\} \backslash\{0\}$.

Remark 1.7. Under assumptions (H0)-(H7), uniform Evans stability $(\mathcal{D})$ implies linearized and nonlinear stability in both the long time and small viscosity limits of standing shocks of symmetric hyperbolic-parabolic systems that are symmetricdissipative at $U_{ \pm}$; see GMWZ4, Z3, N2.

\subsection{Main results.}

1.3.1. Convergence. Denote by $D_{X}(\tilde{\xi}, \lambda)$ the Evans function associated with $\bar{U}^{X}$ and by $D_{-}(\tilde{\xi}, \lambda)$ the Evans function associated with the constant boundary layer $U \equiv U_{-}$at the left-hand endstate $U_{-}$of $\hat{U}$. Then, our first main result is as follows.

Theorem 1.8. Under assumptions (HO)-( $\left.\mathrm{H}^{7}\right)$, there exists a continuous nonvanishing function $\beta(\tilde{\xi}, \lambda, X)$, analytic in $(\tilde{\xi}, \lambda)$, such that

$$
\frac{D_{X}(\tilde{\xi}, \lambda)}{\beta(\tilde{\xi}, \lambda, X)} \rightarrow D_{-}(\tilde{\xi}, \lambda) \mathcal{D}(\tilde{\xi}, \lambda)
$$

as $X \rightarrow \infty$, uniformly on compact subsets of $\left\{\tilde{\xi} \in \mathbb{R}^{d-1}, \Re \lambda \geq 0\right\}$.

Corollary 1.9. Under (HO)-(H7), weak spectral stability of both the constant boundary layer $U \equiv U_{-}$and the standing shock $\hat{U}$ are necessary conditions for stability of $\bar{U}^{X}$ in the standing-shock limit $X \rightarrow \infty$.

Proof. Convergence of zeros of uniformly convergent analytic (in $\lambda$ ) functions.

Remarks 1.10. 1. Under the stronger definition of uniform Evans stability defined in GMWZ5, GMWZ6] involving also a rescaled high-frequency condition, and assuming (H0)-(H7), uniform Evans stability of the constant boundary layer $U \equiv U_{-}$ and the standing shock $\hat{U}$ are also sufficient conditions for spectral stability of $\bar{U}^{X}$ in the standing-shock limit $X \rightarrow \infty$ for frequencies uniformly bounded away from the origin $(\tilde{\xi}, \lambda)=(0,0)$. For intermediate frequencies $R^{-1} \leq|(\tilde{\xi}, \lambda)| \leq R$, this is an immediate consequence of Theorem 1.8. For high frequencies $|(\tilde{\xi}, \lambda)| \geq R, R>0$ sufficiently large, it follows from the fact established in Section 3.2 [GMWZ5, that high-frequency stability is equivalent to stability of the constant layer $U \equiv U_{0}$, and the fact that $U_{0}^{X} \rightarrow U_{-}$as $X \rightarrow \infty$. That is, assuming stability of $\hat{U}$ and $U \equiv U_{-}$, unstable frequencies, if they occur, must converge to the origin as $X \rightarrow \infty$, with no additional assumptions on the system (1.2).

2. It is shown in Corollary 1.29 GMWZ5 that constant boundary layers of symmetric-dissipative systems are uniformly Evans stable (under the stronger definition of [GMWZ5, GMWZ6]) for Dirichlet boundary conditions. Thus, if (1.2) is symmetric-dissipative at $U_{-}$and the boundary conditions are Dirichlet-type, then the constant layer $U \equiv U_{-}$is stable, with the implications above. In the general case, stability or instability of the constant layer $U \equiv U_{-}$may be determined by a linear algebraic computation, since the constant-coefficient eigenvalue ODE is explicitly soluble for each frequency $(\tilde{\xi}, \lambda)$; see [GMWZ5] for further discussion. 
1.3.2. One-dimensional stability. Due to translational invariance, $\mathcal{D}(0,0)=0$, and so we cannot conclude nonvanishing of $D_{X}$ near the origin as $X \rightarrow \infty$ from the convergence result (1.12). Indeed, it is possible that the zero of $\mathcal{D}$ at the origin may perturb into the unstable half-plane $\Re \lambda>0$ for boundary layers with $X$ large, yielding instability. In the one-dimensional setting, this may be detected by the stability index

$$
\Gamma:=\operatorname{sgn} \lim _{\lambda \rightarrow 0^{+} \text {real }} D(0, \lambda) \times \lim _{\lambda \rightarrow+\infty \text { real }} \operatorname{sgn} D(0, \lambda),
$$

where $D$ is chosen with a standard normalization guaranteeing that it is real for real $\lambda$ and $\tilde{\xi}=0$. The stability index is well-defined by the properties that $D$ is continuous along rays at the origin and nonvanishing for real $\lambda$ sufficiently large; see GZ, SZ, Z3. for further discussion. Negativity of $\Gamma$, by the Intermediate Value Theorem, implies existence of a real positive root $D\left(0, \lambda_{*}\right)=0$, hence one-dimensional instability.

Observing that stability of the constant layer $U \equiv U_{-}$and the limiting shock $\hat{U}$ imply that $\operatorname{sgn} D_{-}(\lambda)$ and $\operatorname{sgn} \mathcal{D}(\lambda)$ are constant for real $\lambda \geq 0$, we thus obtain by convergence, (1.12), that a necessary condition for stability in the standing shock limit $X \rightarrow+\infty$, assuming stability of the constant layer, is nonnegativity of

$$
\hat{\Gamma}:=\lim _{X \rightarrow+\infty \text { real }} \frac{D_{X}(0, \lambda)}{D_{-}(0, \lambda)} \times \lim _{\lambda \rightarrow 0^{+} \text {real }} \operatorname{sgn} \mathcal{D}(0, \lambda),
$$

provided these limits exist.

A standard shock stability result [GZ, ZS, [Z3] is that, with appropriate normalization,

$$
\begin{aligned}
\lim _{\lambda \rightarrow 0^{+} \text {real }} \operatorname{sgn} \mathcal{D}(0, \lambda) & =\operatorname{sgn} \partial_{\lambda} \mathcal{D}(0,0)=\operatorname{sgn} \delta, \\
\delta & :=\operatorname{sgn} \operatorname{det}\left(R^{-}, R^{+},[U]\right),
\end{aligned}
$$

where $R^{-}$and $R^{+}$are matrix blocks whose columns span the stable subspace of $d F_{1}\left(U_{-}\right)$and the unstable subspace of $d F_{1}\left(U_{+}\right)$and $[U]:=U_{+}-U_{-}$denotes the jump across the shock. The determinant $\operatorname{det}\left(R^{-}, R^{+},[U]\right)$ may be recognized as the Lopatinski determinant of one-dimensional inviscid theory, whose nonvanishing is equivalent to one-dimensional inviscid shock stability.

Our second main result asserts that the first limit in (1.13) also exists, yielding a necessary stability condition of nonnegativity of a certain Lopatinski-like determinant $\hat{\delta}$ relative to the sign of $\delta$.

Theorem 1.11. Assuming (HO)-(H7) and stability of the constant layer $U \equiv U_{-}$ and the limiting shock $\hat{U}$,

$$
\begin{aligned}
\lim _{X \rightarrow+\infty} \operatorname{sgn} \lim _{\lambda \rightarrow 0^{+} \text {real }} \frac{D_{X}(0, \lambda)}{D_{-}(0, \lambda)} & =\operatorname{sgn} \hat{\delta}, \\
\hat{\delta} & :=\operatorname{sgn} \operatorname{det}\left(\hat{R}^{-}, R_{+}, \hat{V}\right)
\end{aligned}
$$

provided $\hat{\delta} \neq 0$ and $S:=\lim _{z \rightarrow-\infty} \frac{\hat{U}^{\prime}}{\left|\hat{U}^{\prime}\right|}(z)$ exists, where $\hat{R}^{-}$has the dimensions of $R^{-}$and $\hat{V}$ is a single column vector; hence

$$
\operatorname{sgn} \delta \hat{\delta}=\operatorname{sgn} \operatorname{det}\left(R^{-}, R_{+},[U]\right) \operatorname{det}\left(\hat{R}^{-}, R_{+}, \hat{V}\right) \geq 0
$$

is necessary for one-dimensional stability in the standing-shock shock limit. 
For a Lax 1-shock with Dirichlet boundary conditions,

$$
\delta=\operatorname{det}\left(R_{+},[U]\right) \text { and } \hat{\delta}=\operatorname{det}\left(R_{+}, d F_{1}\left(U_{-}\right) S\right)
$$

so that (1.15) becomes

$$
\operatorname{sgn} \operatorname{det}\left(R_{+},[U]\right) \operatorname{det}\left(R_{+}, d F_{1}\left(U_{-}\right) S\right) \geq 0 .
$$

Remarks 1.12. 1. In the (characteristic) limit $U_{-} \rightarrow U_{+}$as the amplitude of the background shock $\hat{U}$ goes to zero, $S \sim[U] \sim r_{1}^{ \pm}$, where $r_{1}$ is the eigenvector of $A_{1}$ associated with the smallest eigenvalue $a_{1}$, and $d F_{1}\left(U_{-}\right) S \sim A_{1}^{-} r_{1}^{-}=a_{1}^{-}[U]$, where $a_{1}^{-}>0$ by the Lax shock conditions (1.7). Thus, $\operatorname{sgn} \operatorname{det}\left(R_{+},[U]\right) \operatorname{det}\left(R_{+}\right.$, $\left.d F_{1}\left(U_{-}\right) S\right) \sim \operatorname{sgn} \operatorname{det}\left(R_{+},[U]\right)^{2}>0$, consistent with stability.

2. From the boundary-layer ODE, $d F_{1}(\hat{U}) \hat{U}^{\prime}=\left(B_{11}(\hat{U}) \hat{U}^{\prime}\right)^{\prime}$, giving $A_{1}\left(x_{1}\right) \hat{U}^{\prime}=$ $B_{11} \hat{U}^{\prime \prime}$, where ' denotes $\partial_{x_{1}}$. Thus, $A_{1}^{-} S=\alpha B_{11}^{-} S$, where $\alpha=\lim _{z \rightarrow-\infty}\left(\hat{U}^{\prime} / \hat{U}\right)(z)$ is necessarily real and positive if the limit $S:=\lim _{z \rightarrow-\infty}\left(\hat{U}^{\prime} /\left|\hat{U}^{\prime}\right|\right)(z)$ exists. It follows that we may replace (1.17) by the equivalent condition

$$
\left.\operatorname{det}\left(R_{+},[U]\right)\right) \operatorname{det}\left(R_{+}, B_{11}\left(U_{-}\right) S\right) \geq 0,
$$

which may be recognized as the necessary condition derived by a rather different argument in Section 4.1 of [SZ].

Our third result states that for Lax 1-shocks the necessary conditions we have derived are also essentially sufficient for one-dimensional stability in the standing shock limit.

Theorem 1.13. For a Lax 1-shock with general boundary conditions (1.6), assuming (H0)-(H7), positivity of $\hat{\Gamma}$ together with stability of the constant layer $U \equiv U_{-}$ and the limiting shock $\hat{U}$ is sufficient for one-dimensional stability in the standingshock limit $X \rightarrow+\infty$.

Remarks 1.14. 1. It was shown in $\mathrm{HuZ}$ that in the limit $U_{-} \rightarrow U_{+}$, the background shock $\hat{U}$ is stable. For symmetric dissipative systems with Dirichlet boundary conditions, the constant layer $U \equiv U_{-}$is stable. By Remark 1.12 1 , therefore, for Lax 1-shocks of symmetric-dissipative systems, with Dirichlet boundary conditions, layers $\hat{U}^{X}$ are one-dimensionally stable in the standing-shock limit $X \rightarrow+\infty$ for $U_{+}$fixed and shock amplitude $\left|U_{+}-U_{-}\right|$sufficiently small.

2. By Remark [1.12,2, the necessary condition of [SZ], together with stability of the limiting shock $\hat{U}^{\prime}$ and the constant layer $U \equiv U_{-}$, is also sufficient for one-dimensional stability. For ideal gas dynamics, the numerical study of HLyZ1] indicates one-dimensional stability of arbitrary shock waves $\hat{U}$ for gas constant $\gamma$ within the physical range $1.2 \leq \gamma \leq 3$ (the only values considered); likewise, $U \equiv U_{-}$ is stable by symmetric dissipativity of the compressible Navier-Stokes equations. Thus, for ideal gas dynamics with Dirichlet boundary conditions and $1.2 \leq \gamma \leq 3$, one-dimensional stability in the standing Lax 1-shock limit is completely decided by the simple algebraic condition (1.18) of [SZ].

For Lax $p$-shocks, $p \geq 2$, the situation is more complicated, involving a tricky double limit. In particular, the conditions of Theorem 1.11 are only necessary and not sufficient for stability. 
1.3.3. Multi-dimensional stability. We restrict now to the case of a Lax 1-shock, for simplicity taking pure Dirichlet boundary conditions, $\operatorname{dim} \Upsilon_{3}=0$. In multidimensions, uniform inviscid stability of a Lax 1-shock is defined as nonvanishing of the multi-dimensional Lopatinski determinant

$$
\Delta(\tilde{\xi}, \lambda):=\operatorname{det}\left(\mathcal{R}_{+}(\tilde{\xi}, \lambda), \lambda[U]+\sum_{j=2}^{d} i \xi_{j}\left[F_{j}(U)\right]\right)
$$

on the nonnegative unit sphere $\mathcal{S}^{+}:=\{|(\tilde{\xi}, \lambda)|=1, \Re \lambda \geq 0\}$, where $\mathcal{R}_{+}$is a matrix block whose columns form a basis for the unstable subspaces of

$$
\mathcal{A}_{+}(\tilde{\xi}, \lambda):=\left(\lambda I+\sum_{j=2}^{d} i \xi_{j} d F_{j}\left(U_{+}\right)\right) d F_{1}\left(U_{+}\right)^{-1}
$$

and $[h(U)]:=h\left(U_{+}\right)-h\left(U_{-}\right)$denotes a jump in $h$ across the shock. Define the related determinant

$$
\hat{\Delta}(\tilde{\xi}, \lambda, \eta):=\operatorname{det}\left(\mathcal{R}_{+}(\tilde{\xi}, \lambda), \lambda[U]+\sum_{j=2}^{d} i \xi_{j}\left[F_{j}(U)\right]+\eta d F_{1}\left(U_{-}\right) S_{-}\right),
$$

$\eta \in \mathbb{R}$, where $S:=\lim _{z \rightarrow-\infty} \frac{\hat{U}^{\prime}}{\left|\hat{U}^{\prime}\right|}(z)$. Then, our fourth and fifth main results, giving necessary conditions and sufficient conditions for multi-dimensional stability analogous to those of Theorems 1.11 and 1.13, are as follows.

Theorem 1.15. For a Lax 1-shock and Dirichlet boundary conditions, assuming (HO)-(H7), a necessary condition for stability of $\bar{U}_{X}$ in the standing-shock limit $X \rightarrow+\infty$ is that $\hat{\Delta}$ have no root that is simple with respect to $\lambda$ on the positive half-sphere

$$
\hat{\mathcal{S}}^{+}:=\{|\tilde{\xi}, \lambda, \eta|=1, \Re \lambda>0, \eta>0\},
$$

in the sense that $\hat{\Delta}=0$ and $\partial_{\lambda} \hat{\Delta} \neq 0$.

Theorem 1.16. For a Lax 1-shock and Dirichlet boundary conditions, assuming (H0)-(H7), sufficient conditions for stability of $\bar{U}_{X}$ in the standing-shock limit $X \rightarrow$ $+\infty$ are the nonvanishing of $\hat{\Delta}$ on the nonnegative half-sphere $\hat{\mathcal{S}}^{+}:=\{|\tilde{\xi}, \lambda, \eta|=$ $1, \Re \lambda \geq 0, \eta \geq 0\}$, stability of the limiting shock $\hat{U}$, and stability of the constant layer $U \equiv U_{-}$,

Remark 1.17. In the one-dimensional case $\tilde{\xi} \equiv 0, \mathcal{R}_{ \pm} \equiv R_{ \pm}=$constant, so that $\partial_{\lambda} \hat{\Delta} \equiv \delta$. It is readily seen that Theorems 1.15 and 1.16 reduce in this context to the restrictions of Theorems 1.11 and 1.13 to the case of a Lax 1-shock and Dirichlet boundary conditions, provided that the limiting shock $\hat{U}$ is one-dimensionally stable, so that $\delta \neq 0[\mathrm{ZH}]$.

Remarks 1.18. 1. Multi-dimensional stability of shock waves in the limit $U_{-} \rightarrow$ $U_{+}$has been established in FS for symmetric dissipative systems with strictly parabolic (Laplacian) viscosity $\sum\left(B_{j k} U_{x_{j}}\right)_{x_{k}}$. By the arguments of Remarks 1.121 and 1.14 1 , therefore, boundary layers of such systems with Dirichlet boundary conditions are multi-dimensionally stable in the standing Lax 1-shock limit for $U_{+}$ fixed and $\left|U_{+}-U_{-}\right|$sufficiently small. The argument of [FS] appears likely to generalize to the general symmetric dissipative case (see also [PZ]), which would extend the boundary-layer result also to the general case. 
2. For ideal gas dynamics, the numerical study of HLyZ2 indicates multidimensional stability of arbitrary shock waves for gas constants in the physical range $1.2 \leq \gamma \leq 3$. By the arguments of Remarks 1.122 and 1.14,2, therefore, for ideal gas dynamics with Dirichlet boundary conditions and $1.2 \leq \gamma \leq 3$, multi-dimensional stability in the standing Lax 1-shock limit is completely decided by vanishing or nonvanishing of the extended Lopatinski determinant $\hat{\Delta}$ defined in (1.21), a simple, linear-algebraic condition.

1.3.4. Verification. Evidently, the nonvanishing of $\hat{\Delta}$ on the nonnegative half-sphere in $(\tilde{\xi}, \lambda, \eta)$ is equivalent to the condition that the image of

$$
\hat{\eta}(\tilde{\xi}, \lambda):=-\Delta(\tilde{\xi}, \lambda) / \operatorname{det}\left(\mathcal{R}_{+}(\tilde{\xi}, \lambda), d F_{1}\left(U_{-}\right) S_{-}\right)
$$

over the nonnegative half-sphere in $(\tilde{\xi}, \lambda)$ avoid the nonnegative real axis, a natural generalization of the one-dimensional condition (1.17). Note that $\hat{\eta}$ is independent of the choice of $\mathcal{R}_{+}$and is homogeneous of degree one in $(\tilde{\xi}, \lambda)$. This leads us to the following condition convenient for numerical or analytic verification.

Proposition 1.19. Assuming the one-dimensional stability condition (1.17), the nonvanishing of $\hat{\Delta}$ on the nonnegative half-sphere in $(\tilde{\xi}, \lambda, \eta)$ is equivalent to the condition that the image of $\hat{\eta}(1, i \tau)$ over $\tau \in \mathbb{R}$, with $\hat{\eta}$ defined as in (1.22), does not intersect the nonnegative real axis.

Proof. By homogeneity of $\hat{\Delta}$, nonvanishing on the half-sphere is equivalent to nonvanishing of $\hat{\delta}(0, \lambda, \eta)$, or (1.17), and nonvanishing of $\hat{\delta}(1, \lambda, \eta)$, or the condition that $\hat{\eta}(1, \lambda)$ avoid the nonnegative real axis for $\Re \lambda \geq 0$. Recalling the standard fact that $\mathcal{R}_{+}(1, \lambda)$, hence $\hat{\eta}(1, \lambda)$, may be chosen to be analytic in $\lambda$ for $\Re \lambda>0$ and continuous at $\Re \lambda=0$, we find by the argument principle applied to a sufficiently large semicircle about the origin, bounded to the left by the imaginary axis, and the fact that $\hat{\eta}(1, \lambda)$ by homogeneity/continuity does not intersect the nonnegative real axis for $\Re \lambda \geq 0$ and $|\lambda|$ sufficiently large (by the assumed one-dimensional stability) that the latter condition is equivalent to nonintersection with the nonnegative real axis as $\lambda$ traverses the imaginary axis.

1.4. Discusssion and open problems. The results of Theorems 1.15 and 1.16 illuminate and greatly extend the earlier results of [SZ] and [CHNZ] in the onedimensional case. In particular, we regard the derivation of necessary and sufficient conditions for multi-dimensional stability as a substantial advance. Though our necessary and our sufficient conditions are slightly different, the difference is sufficiently slight that it should not interfere in practice with the classification of physical stability regions.

We note that, besides its independent interest, the treatment of the standing shock limit, as pointed out in [CHNZ, is important in truncating the computational domain for global stability analyses.

On the other hand, we have restricted here mainly to the simplest case of a Lax 1-shock, which corresponds to the case of inflow boundary conditions. It would be very interesting to obtain corresponding conditions also in the case of outflow boundary conditions, for example, a Lax $n$-shock. Likewise, it would be very interesting to carry out computations analogous to those carried out for gas dynamics in Section 5 also for the equations of MHD. 


\section{Construction of the Evans function}

We begin by reviewing the construction of the Evans function following [Z3, GMWZ5, GMWZ6, NZ1, [NZ2,

2.1. Expression as a first-order system. We first observe that the matrix

$$
\left(\begin{array}{cc}
A_{1}^{11} & A_{1}^{12} \\
B_{11}^{21} & B_{11}^{22}
\end{array}\right)=\left(\begin{array}{cc}
d F_{1}^{11} & d F_{1}^{12} \\
B_{11}^{21} & B_{11}^{22}
\end{array}\right)
$$

is full rank, by (H2)-(H3) together with the block structure assumption (1.4), as can be seen most easily by working in $\tilde{W}$-coordinates; see [Z1, Z3, MaZ3].

As a consequence, the Fourier-transformed eigenvalue equations (1.10), (1.11) may be written as a first-order system

$$
\begin{gathered}
Z^{\prime}=\mathbb{G}_{X}\left(\tilde{\xi}, \lambda, x_{1}\right) Z, \\
M(\tilde{\xi}) Z=0
\end{gathered}
$$

in the convenient coordinates

$$
Z=\left(\begin{array}{c}
Z_{1} \\
Z_{2} \\
Z_{3}
\end{array}\right):=\left(\begin{array}{c}
B_{11}^{21} u+B_{11}^{22} v \\
B_{11} U^{\prime}-A_{1} U
\end{array}\right)=\left(\begin{array}{c}
B_{11}^{21} u+B_{11}^{22} v \\
-A_{1}^{11} u-A_{1}^{12} v \\
B_{11}^{21} u^{\prime}+B_{11}^{22} v^{\prime}-A_{1}^{21} u-A_{1}^{22} v
\end{array}\right)
$$

see [Z3] for a further discussion.

In the case of Dirichlet boundary conditions $\operatorname{dim} \Upsilon_{3}=0$, the boundary operator $M$ is independent of $\tilde{\xi}$, with

$$
\operatorname{ker} M=\left\{Z: Z_{1}=0, Z_{2}=0\right\}
$$

in the inflow case and

$$
\operatorname{ker} M=\left\{Z: Z_{1}=0\right\}
$$

in the outflow case. In general, $\operatorname{ker} B(\tilde{\xi})$ depends on $\tilde{\xi}$ in a possibly complicated way. By the underlying relation $\bar{U}_{X}\left(x_{1}\right)=\hat{U}\left(x_{1}-X\right)$, we have

$$
\mathbb{G}_{X}\left(\tilde{\xi}, \lambda, x_{1}\right)=\mathbb{G}\left(\tilde{\xi}, \lambda, x_{1}-X\right) .
$$

2.2. Conjugation to constant coefficients. We next recall the following result, established in [Z3, GMWZ5, a consequence of the conjugation lemma introduced in [MZ1] and the fact proved in MaZ3, Z3, that $\hat{U}$ under hypotheses (H0)-(H7) converges exponentially to $U_{ \pm}$as $x_{1} \rightarrow \pm \infty$. See, e.g., [Z3] for details.

Proposition 2.1. There exist matrix-valued functions

$$
T_{X}^{ \pm}\left(\tilde{\xi}, \lambda, x_{1}\right)=T^{ \pm}\left(\tilde{\xi}, \lambda, x_{1}-X\right),
$$

uniformly bounded with bounded inverse for $x_{1} \gtrless 0$, locally analytic in $(\tilde{\xi}, \lambda)$, such that

$$
\left|\left(T^{ \pm}-I d\right)\left(x_{1}\right)\right| \leq C e^{-\theta\left|x_{1}\right|} \text { for } x_{1} \gtrless 0,
$$

$\theta>0$, and $Z:=T_{X}^{ \pm} X$ satisfies the constant-coefficient equation

$$
X^{\prime}=\mathbb{G}_{X}^{ \pm}(\tilde{\xi}, \lambda) X \text { for } x_{1} \gtrless X,
$$

$\mathbb{G}_{X}^{ \pm}(\tilde{\xi}, \lambda):=\mathbb{G}_{X}^{ \pm}(\tilde{\xi}, \lambda, \pm \infty)$, whenever $Z$ satisfies (2.1). 
2.3. Definition of the Evans function. Finally, we recall the following standard result, established in increasing generality in [SZ, Z1, Z3, GMWZ4, GMWZ6].

Proposition 2.2. Under (HO)-(H7), there exist matrices $\mathbb{E}^{-}, \mathbb{E}^{+}$, and $\mathbb{E}^{0}$ whose columns form bases of the unstable subspace of $\mathbb{G}^{-}$, the stable subspace of $\mathbb{G}^{+}$, and $\operatorname{ker} \mathcal{M}(\tilde{\xi})$ and matrices $\mathbb{F}^{-}$and $\mathbb{F}^{+}$whose columns form bases of the the stable subspace of $\mathbb{G}^{-}$and the unstable subspace of $\mathbb{G}^{+}, C^{\infty}$ on $\{\Re \lambda \geq 0\} \backslash\{(0,0)\}$ and continuously extendable along rays through the origin. Moreover, $\mathbb{E}^{+}$and $\mathbb{F}^{+}$and $\mathbb{E}^{-}$and $\mathbb{F}^{-}$are uniformly transverse on compact subsets of $\{\Re \lambda \geq 0\}$ and

$$
\operatorname{dim} \operatorname{Span} \mathbb{E}^{-}=\operatorname{dim} \operatorname{Span} \mathbb{E}^{0}=(n+r)-\operatorname{dim} \operatorname{Span} \mathbb{E}^{-} .
$$

Remark 2.3. In the one-dimensional setting, the subspaces of Proposition 2.2 may be defined as globally analytic functions on $\Re \lambda \geq-\eta, \eta>0$, using a standard construction of Kato [Kat]; see, e.g., GZ, Z3, CHNZ].

With these preparations, we define the shock Evans function precisely as the $(n+r) \times(n+r)$ Wronskian

$$
\mathcal{D}(\tilde{\xi}, \lambda):=\left.\operatorname{det}\left(T^{-} \mathbb{E}^{-}, T^{+} \mathbb{E}^{+}\right)\right|_{x_{1}=0}
$$

and the boundary-layer Evans function as

$$
D_{X}(\tilde{\xi}, \lambda):=\left.\operatorname{det}\left(\mathbb{E}^{0}, T_{X}^{+} \mathbb{E}^{+}\right)\right|_{x_{1}=0}=\left.\operatorname{det}\left(\mathbb{E}^{0}, T^{+} \mathbb{E}^{+}\right)\right|_{x_{1}=-X} .
$$

The Evans function for the constant layer $U \equiv U_{-}$is given by

$$
D_{-}(\tilde{\xi}, \lambda):=\operatorname{det}\left(\mathbb{E}^{0}, \mathbb{F}^{-}\right),
$$

since in this case $\mathbb{E}^{+}=\mathbb{F}^{-}$, or equivalently by

$$
D_{-}(\tilde{\xi}, \lambda):=\operatorname{det}\left(\tilde{\mathbb{E}}^{-*} \mathbb{E}^{0}\right),
$$

where

$$
\left(\tilde{\mathbb{E}}^{-}, \tilde{\mathbb{F}}^{-}\right)=\left(\mathbb{E}^{-}, \mathbb{F}^{-}\right)^{-1 *}
$$

are dual bases to $\left(\mathbb{E}^{-}, \mathbb{F}^{-}\right)$with respect to the standard complex inner product, $M^{*}$ denoting the adjoint, or conjugate transpose, of a matrix $M$.

2.4. Behavior near zero. For later use, we record the following refinement of Proposition 2.2 (also established in [SZ, Z1, Z3, GMWZ4, GMWZ6]), from which we may determine the behavior of $\mathcal{D}, D_{x}$ as $(\tilde{\xi}, \lambda) \rightarrow(0,0)$.

Proposition 2.4. Under (HO) $-\left(H^{7}\right)$, the limiting subspaces

$$
\lim _{\rho \rightarrow 0^{+}} \operatorname{Span} T^{+} e^{\mathbb{G}^{+} x_{1}} \mathbb{E}^{+}\left(\rho \tilde{\xi}_{0}, \rho \lambda_{0}\right) \text { and } \lim _{\rho \rightarrow 0^{+}} \operatorname{Span} T^{-} e^{\mathbb{G}^{-} x_{1}} \mathbb{E}^{-}\left(\rho \tilde{\xi}_{0}, \rho \lambda_{0}\right),
$$

$\rho \in \mathbb{R}, \Re \lambda_{0} \geq 0$, are spanned by the direct sum of fast modes

$$
Z_{1}=b_{11} \phi, \quad\left(Z_{2}, Z_{3}\right)=0,
$$

with $\phi$ satisfying $B_{11} \phi^{\prime}-A_{1} \phi=0$ and decaying at $+\infty$ [resp. $-\infty$ ] and slow modes

$$
Z_{1}=*, \quad\left(Z_{2}, Z_{3}\right)=\mathcal{R},
$$

with $\mathcal{R}$ spanning the unstable [resp. stable] subspace of $\mathcal{A}_{+}$[resp. $\mathcal{A}_{-}$], with

$$
\mathcal{A}_{ \pm}(\tilde{\xi}, \lambda):=\left(\lambda I+\sum_{j=2}^{d} i \xi_{j} d F_{j}\left(U_{ \pm}\right)\right) d F_{1}\left(U_{ \pm}\right)^{-1}
$$


expressible alternatively as $Z=T^{ \pm} X$ with

$$
X_{1}=b_{11}\left(-A_{1}^{ \pm}\right)^{-1} \mathcal{R}, \quad\left(X_{2}, X_{3}\right)=\mathcal{R}
$$

constant. Symmetric decompositions hold for $T^{+} e^{\mathbb{G}^{+} x_{1}} \mathbb{F}^{+}$and $T^{-} e^{\mathbb{G}^{-} x_{1}} \mathbb{F}^{-}$.

\section{BASIC CONVERGENCE RESUlT}

Proof of Theorem 1.8 for $(\tilde{\xi}, \lambda)$ bounded away from the origin. Viewing $\mathcal{D}$ and $D_{X}$ as Wronskians of solutions of the same ODE (2.1), we may rewrite (2.9) using Abel's Theorem as

$$
D_{X}(\tilde{\xi}, \lambda):=\left.e^{\int_{0}^{-X} \operatorname{Trace} \mathbb{G}(\tilde{\xi}, \lambda, z) d z} \operatorname{det}\left(\mathcal{S}^{-X \rightarrow 0} \mathbb{E}^{0}, T^{+} \mathbb{E}^{+}\right)\right|_{x_{1}=0},
$$

where $\mathcal{S}^{y \rightarrow x}$ denotes the solution operator of (2.1).

Next, expand

$$
\begin{aligned}
\mathbb{E}^{0} & =T^{-}(-X) T^{-}(-X)^{-1} \mathbb{E}^{0} \\
& =\left.T^{-} \mathbb{E}^{0}\right|_{x_{1}=-X}+O\left(e^{-\theta X}\right) \\
& =\left.T^{-} \Pi_{\mathbb{E}^{-}} \mathbb{E}^{0}\right|_{x_{1}=-X}+\left.T^{-} \Pi_{\mathbb{F}^{-}} \mathbb{E}^{0}\right|_{x_{1}=-X}+O\left(e^{-\theta X}\right),
\end{aligned}
$$

where

$$
\Pi_{\mathbb{E}^{-}}=\mathbb{E}^{-} \tilde{\mathbb{E}}^{-*} \text { and } \Pi_{\mathbb{F}^{-}}=\mathbb{F}^{-\tilde{\mathbb{F}}^{-*}}
$$

denote the eigenprojections of $\mathbb{G}^{-}$onto subspaces $\mathbb{E}^{-}$and $\mathbb{F}^{-}$, noting that

$$
T^{-} \Pi_{\mathbb{E}^{-}} \mathbb{E}^{0}=\left(T^{-} \mathbb{E}^{-}\right)\left(\tilde{\mathbb{E}}^{-*} \mathbb{E}^{0}\right) .
$$

From (3.4), we obtain

$$
\begin{aligned}
& \left.\operatorname{det}\left(\mathcal{S}^{-X \rightarrow 0} T^{-} \Pi_{\mathbb{E}^{-}} \mathbb{E}^{0}, T^{+} \mathbb{E}^{+}\right)\right|_{x_{1}=0} \\
& \quad=\left.\operatorname{det}\left(\mathcal{S}^{-X \rightarrow 0} T^{-} \mathbb{E}^{-}, T^{+} \mathbb{E}^{+}\right)\right|_{x_{1}=0} \operatorname{det}\left(\tilde{\mathbb{E}}^{-*} \mathbb{E}^{0}\right) \\
& \quad=\left.\operatorname{det}\left(\mathcal{S}^{-X \rightarrow 0} T^{-} \mathbb{E}^{-}, T^{+} \mathbb{E}^{+}\right)\right|_{x_{1}=0} D_{-} .
\end{aligned}
$$

Next, expanding

$$
\mathcal{S}^{y \rightarrow x} T^{-}(y)=T^{-}(x) e^{\mathbb{G}^{-}(x-y)}
$$

and noting that

$$
e^{\mathbb{G}^{-}(x-y)} \mathbb{E}^{-}=e^{\Pi_{\mathbb{E}^{-}} \mathbb{G}^{-}(x-y)} \Pi_{\mathbb{E}^{-}} \mathbb{E}^{-}=\mathbb{E}^{-} e^{\tilde{\mathbb{E}}^{-*} \mathbb{G}^{-} \mathbb{E}^{-}(x-y)},
$$

where Trace $\left(\tilde{\mathbb{E}}^{-*} \mathbb{G}^{-} \mathbb{E}^{-}\right)=\operatorname{Trace}\left(\tilde{\mathbb{E}}^{-*} \mathbb{E}^{-} \mathbb{G}^{-}\right)=$Trace $\Pi_{\mathbb{E}^{-}} \mathbb{G}^{-}$, we find that

$$
\begin{aligned}
\left.\operatorname{det}\left(\mathcal{S}^{-X \rightarrow 0} T^{-} \mathbb{E}^{-}, T^{+} \mathbb{E}^{+}\right)\right|_{x_{1}=0} \\
=\left.e^{\operatorname{Trace}\left(\Pi_{\mathbb{E}^{-}} \mathbb{G}^{-}\right) X} \operatorname{det}\left(T^{-} \mathbb{E}^{-}, T^{+} \mathbb{E}^{+}\right)\right|_{x_{1}=0} \\
=e^{\operatorname{Trace}\left(\Pi_{\mathbb{E}^{-}} \mathbb{G}^{-}\right) X} \mathcal{D},
\end{aligned}
$$

where, since $\mathbb{E}^{-}$is the unstable subspace of $\mathbb{G}^{-}$,

$$
e^{\operatorname{Trace}\left(\Pi_{\mathbb{E}^{-}} \mathbb{G}^{-}\right) X}
$$

is uniformly exponentially growing in $X$ for $\Re \lambda \geq 0$. Indeed, for $(\tilde{\xi}, \lambda)$ bounded from the origin $\Re \lambda \geq 0$, each column of $\mathcal{S}^{-X \rightarrow 0} T^{-} \mathbb{E}^{-}$is uniformly exponentially growing in $X$ at a rate at least

$$
e^{\mu_{*} X},
$$


where $\mu_{*}(\tilde{\xi}, \lambda)$ is the smallest real part of the (positive real part) eigenvalues of $\mathbb{G}^{-}$ associated with the unstable subspace $\mathbb{E}^{-}$.

By a similar argument, each column of $\mathcal{S}^{-X \rightarrow 0} T^{-} \Pi_{\mathbb{F}^{-}} \mathbb{E}^{0}$ is uniformly exponentially decaying in $X$, at a rate $e^{\mu^{*} X}$, where $\mu^{*}$ is the largest real part of the (negative real part) eigenvalues of $\mathbb{G}^{-}$associated with the stable subspace $\mathbb{F}^{-}$. Collecting information, we thus have

$$
\begin{aligned}
\frac{D_{X}(\tilde{\xi}, \lambda)}{\beta(\tilde{\xi}, \lambda, X)} & =\left(D_{-}(\tilde{\xi}, \lambda) \mathcal{D}(\tilde{\xi}, \lambda)+O\left(e^{-\theta X}\right)+O\left(e^{\left(\mu_{*}-\mu^{*}\right) X}\right)\right) \\
& \rightarrow D_{-}(\tilde{\xi}, \lambda) \mathcal{D}(\tilde{\xi}, \lambda)
\end{aligned}
$$

as $X \rightarrow+\infty$, exponentially in $X$, where

$$
\beta(\tilde{\xi}, \lambda, X):=e^{\int_{0}^{-X} \operatorname{Trace} \mathbb{G}(\tilde{\xi}, \lambda, z) d z} e^{\operatorname{Trace}\left(\Pi_{\mathbb{E}^{-}} \mathbb{G}^{-}\right) X},
$$

for $(\tilde{\xi}, \lambda)$ uniformly bounded away from the origin on $\Re \lambda \geq 0$.

Remark 3.1. In general, the real parts of the eigenvalues of $\mathbb{E}^{-}$and $\mathbb{F}^{-}$can converge to zero as $(\tilde{\xi}, \lambda)$ approaches the origin, so that $\mu_{*}, \mu^{*} \rightarrow 0$ and the above convergence argument fails. In the special case of a Lax 1 -shock, $\mu^{*} \rightarrow 0$, but $\mu_{*}$ remains strictly negative [Z3, and so we obtain uniform convergence by this argument on all of $\Re \lambda \geq 0$. Indeed, in the one-dimensional case, we obtain uniform convergence on $\Re \lambda \geq-\eta, \eta>0$. (The only obstruction in the multi-dimensional case is that $\mathcal{D}$ is not defined on this set [Z3].)

Combining Remarks 1.10, 2.3. and 3.1, we obtain the following simple result, reducing determination of one-dimensional stability to computation of the stability index.

Lemma 3.2. Under (HO)-(H7) and stability of the constant layer $U \equiv U_{-}$, for a stable Lax 1-shock in one dimension, $D_{X}$ has exactly one zero on $\Re \lambda>-\eta, \eta>0$, for $X$ sufficiently large, hence is stable if and only if its stability index is positive.

Proof. By Remarks 1.10, under stability of $U \equiv U_{-}$, we may restrict attention to a compact set of frequencies, while by Remark 2.3 we may take $D_{X}$ and $\mathcal{D}$ analytic on $\Re \lambda \geq-\eta$. As the uniform limit of analytic functions, we find that the number of zeros of $\mathcal{D} D_{-}$on $\Re \lambda>-\eta$ is equal to the number of zeros of $D_{X}$ for $X$ sufficiently large. As the number of zeros of $\mathcal{D}$ is one and the number of zeros of $D_{-}$is zero, by stability, the number of zeros of $D_{X}$ is one as asserted.

As the stability index $\Gamma$ counts the parity of the number of nonstable roots $\Re \lambda>0$ (see [GZ, Z3] ), positivity of $\Gamma$ corresponding to an even number of nonstable roots, and since $\Gamma=0$ corresponds to instability [NZ1], we thus obtain stability if and only if $\Gamma>0$.

\section{BEHAVIOR NEAR THE ORIGIN}

Proof of Theorem 1.8 for $(\tilde{\xi}, \lambda) \rightarrow(0,0)$. The shock Evans function $\mathcal{D}$ is continuous at the origin, with $\mathcal{D}(0,0)=0$, and $D_{-}$is bounded on compact sets. Thus, to complete the proof of Theorem 1.8, it suffices to show that

$$
D_{X}(\tilde{\xi}, \lambda) / \beta(\tilde{\xi}, \lambda) \rightarrow 0
$$

as $(\tilde{\xi}, \lambda) \rightarrow 0$ and $X \rightarrow+\infty$, with $\beta$ defined as in (3.9). 
Noting that $T^{-} \mathbb{E}^{-}(0,0)$ contains by continuity all exponentially decaying solutions of the one-dimensional eigenvalue equation, hence, in particular, $\hat{U}^{\prime}\left(x_{1}\right)$, we may without loss of generality assign the value $\hat{U}^{\prime}\left(x_{1}\right)$ to the first column of $T^{-} \mathbb{E}^{-}$ at $(0,0)$. Moreover, noting that the strongly unstable subspace of $\mathbb{G}^{-}$, defined as the part whose eigenvalues have strictly positive real part even at $(0,0)$, perturbs analytically, we may restrict the first column to this subspace, ensuring that the first column of $\mathcal{S}^{-X \rightarrow 0} T^{-} \mathbb{E}^{-}$is analytic up to the origin and moreover grows exponentially in $X$, at a rate $e^{\theta X}$, for some $\theta>0$ and $|(\tilde{\xi}, \lambda)|$ sufficiently small; hence contributions to $D_{X}$ coming from the first columns of the second two terms in the last line of (3.2) are exponentially small as $X \rightarrow 0$ and can be ignored, while the contributions in other columns are at least bounded. Likewise, we may arrange that the first column of $T^{+} \mathbb{E}^{+}$be analytic up to the origin and equal to $\hat{U}^{\prime}$ at $(\tilde{\xi}, \lambda)=(0,0)$.

In place of (3.8), therefore, we obtain the weaker estimate

$$
\begin{aligned}
\left|D_{X}(\tilde{\xi}, \lambda)\right| / \beta(\tilde{\xi}, \lambda) \leq & \left|D_{X}(\tilde{\xi}, \lambda) \operatorname{det}\left(T^{-}\left(\mathbb{E}_{1}^{-}, O(1)\right), T^{+} \mathbb{E}^{+}\right)_{x_{1}=0}\right| \\
& +O\left(e^{-\theta X}\right)+O\left(e^{\left(\mu_{*}-\mu^{*}\right) X}\right) \\
& \rightarrow 0
\end{aligned}
$$

as $(\tilde{\xi}, \lambda) \rightarrow(0,0), X \rightarrow+\infty$, where $T^{-} \mathbb{E}_{1}^{-}$and $T^{+} \mathbb{E}_{1}^{+}$denote the first columns of $T^{-} \mathbb{E}^{-}$and $T^{+} \mathbb{E}^{+}$, since by our choice of normalization, $T^{-} \mathbb{E}_{1}^{-}$and $T^{+} \mathbb{E}_{1}^{+}$are continuous (indeed, analytic) at the origin and coincide for $(\tilde{\xi}, \lambda)=(0,0)$. This completes the proof of the theorem.

Proof of Theorem 1.11. Fix $\tilde{\xi} \equiv 0$, so that $D(0, \lambda)$ is continuous in $\lambda$. Without loss of generality, take $D_{-}(0,0)=1$, with, moreover,

$$
\mathbb{E}^{0}(0,0) e^{-\tilde{\mathbb{E}}^{-*} \mathbb{G}^{-} \mathbb{E}^{-} X}=T(-X) e^{-\mathbb{G}^{-} X}\left(\mathbb{E}^{-}(0,0)+\mathbb{F}^{-}(0,0) \alpha\right)
$$

for some $k \times k$ matrix $\alpha$, where $k=\operatorname{dim} \mathbb{F}^{-}$. Thus,

$$
\begin{aligned}
\mathcal{S}^{-X \rightarrow 0} \mathbb{E}^{0} & =T^{-}(0)\left(e^{\mathbb{G}^{-} X} T^{-}(-X)^{-1} \mathbb{E}^{0}\right) \\
& =T^{-}(0)\left(\mathbb{E}^{-}(0,0)+\mathbb{F}^{-}(0,0) \alpha\right) e^{-\tilde{\mathbb{E}}^{-*} \mathbb{G}^{-} \mathbb{E}^{-} X} .
\end{aligned}
$$

Following the proof of Theorem 1.8, we find that the quantity

$$
D_{X}(0,0) / e^{\int_{0}^{-X} \operatorname{Trace} \mathbb{G}(0,0, z) d z} e^{\operatorname{Trace}_{\mathbb{E}^{-}} \mathbb{G}^{-} X}
$$

is given (exactly, with no exponentially decaying error) by

$$
\operatorname{det}\left(\left.T^{-}\left(\mathbb{E}^{-}+\mathbb{F}^{-} \alpha, T^{+} \mathbb{E}^{+}\right)\right|_{x_{1}=0,(\tilde{\xi}, \lambda)=(0,0)},\right.
$$

where $\operatorname{Trace} \mathbb{G}(0,0, z)$ and $\operatorname{Trace} \Pi_{E E^{-}} \mathbb{G}^{-}(0,0)$ are real; hence

$$
e^{\int_{0}^{-X} \operatorname{Trace} \mathbb{G}(0,0, z) d z} \text { and } e^{\operatorname{Trace} \Pi_{\mathbb{E}^{-}} \mathbb{G}^{-} X}
$$

are real and positive.

Appealing to Proposition 2.4, we may arrange without loss of generality that the first $k_{1}$ columns of $T^{-}\left(0,0, x_{1}\right) e^{\mathbb{G}^{-}(0,0) x_{1}} \mathbb{E}^{-}(0,0)$ consist of functions $\left(b_{11} \phi_{j}, 0\right)$, where $\phi_{j}, j=1, \ldots, k_{1}$, are solutions of

$$
B_{11} \phi_{j}^{\prime}-A_{1} \phi_{j}=0
$$


that are uniformly exponentially decaying as $x_{1} \rightarrow-\infty$, hence exponentially growing in the forward direction, and the remaining $k_{2}$ columns consist of functions $\left(*, r_{j}^{-}\right), j=1, \ldots, k_{2}$, where $r_{j}^{-}$are constant eigenvectors of $A_{1}^{-}$with negative eigenvalues $a_{j}$, and similarly for $T^{+} \mathbb{E}^{+}$. Likewise, we may arrange that the columns of $T^{-} \mathbb{F}^{-}$consist of $l_{1}$ solutions of (4.4) that are uniformly exponentially decaying in $x_{1}$ in the forward direction and $l_{2}$ functions $\left(*, r_{j}^{-}\right), j=1, \ldots, l_{2}$, where $r_{j}^{-}$are constant eigenvectors of $A_{1}^{-}$with positive eigenvalues $a_{j}$.

Finally, we may choose the first column of $\left.T^{-} \mathbb{E}^{-}\right|_{x_{1}=0}$ as $\hat{U}^{\prime}(0)$, noting that $\hat{U}^{\prime}(0)$ lies also in $\left.T^{+} \mathbb{E}^{+}\right|_{x_{1}=0}$. Combining these facts, we find that, up to an exponentially decaying error with respect to $X$, we may rewrite

$$
D_{X}(0,0) / e^{\int_{0}^{-X} \operatorname{Trace} \mathbb{G}(0,0, z) d z} e^{\operatorname{Trace}\left(\Pi_{\mathbb{E}^{-}} \mathbb{G}^{-}\right) X}
$$

as

$$
\operatorname{det}\left(\begin{array}{ccccccccc}
* & \psi_{2} & \cdots & \psi_{k_{2}} & * & * & \psi_{k_{1}+1} & \cdots & \psi_{n+1} \\
\left|\hat{U}^{\prime}(-X)\right| \hat{V}_{X} & 0 & \cdots & 0 & \hat{\mathcal{R}}^{-} & \mathcal{R}^{+} & 0 & \cdots & 0
\end{array}\right)
$$

evaluated at $x_{1}=0,(\tilde{\xi}, \lambda)=(0,0)$, where $\psi_{j}:=b_{11} \phi_{j},\left(\begin{array}{c}* \\ \left|\hat{U}^{\prime}(-X)\right| \hat{V}_{X}\end{array}\right)$ is the part of the first column of $\mathbb{F}^{-} \alpha$ involving only slow modes

$$
\left(\left(-a_{j}^{-}\right)^{-1} b_{11} r_{j}^{-}, r_{j}^{-}\right)
$$

(recall (2.11) $)$, and $\left(\begin{array}{c}* \\ \hat{\mathcal{R}}^{-}\end{array}\right)$is the part involving only slow modes of the block of $\mathbb{E}^{-}+\mathbb{F}^{-} \alpha$ corresponding to the slow block $\left(\begin{array}{c}* \\ \mathcal{R}^{-}\end{array}\right)$of $\mathbb{E}^{-}$. Referring to (4.2), we see that $\hat{V}_{X}$ has a limit $\hat{V}$ as $X \rightarrow+\infty$ so long as $S:=\lim _{z \rightarrow-\infty}\left(\hat{U}^{\prime} /\left|\hat{U}^{\prime}\right|\right)(z)$ exists (the first column of $T^{-}(-X) e^{-\mathbb{G}^{-} X} \mathbb{E}^{-}$being then approximately $\left|\hat{U}^{\prime}(-X)\right| S$ as $X \rightarrow+\infty$, so that the slow component of the first column of $\mathbb{F}^{-} \alpha$ is approximately $\left|\hat{U}^{\prime}(-X)\right| \hat{V}$ for a fixed $\hat{V}$ determined by $\left.S\right)$.

By a block determinant expansion, we have, therefore,

$$
\begin{aligned}
& D_{X}(0,0) / e^{\int_{0}^{-X} \operatorname{Trace} \mathbb{G}(0,0, z) d z} e^{\operatorname{Trace}\left(\Pi_{\mathbb{E}^{-}} \mathbb{G}^{-}\right) X} \\
& =\left.\sigma\left|\hat{U}^{\prime}(-X)\right| \operatorname{det}\left(\psi_{2}, \ldots, \psi_{n+1}\right) \operatorname{det}\left(\hat{\mathcal{R}}^{-}, \mathcal{R}^{+}, \hat{V}_{X}\right)\right|_{x_{1}=0,(\tilde{\xi}, \lambda)=(0,0)} \\
& \left.\rightarrow \sigma\left|\hat{U}^{\prime}(-X)\right| \operatorname{det}\left(\psi_{2}, \ldots, \psi_{n+1}\right) \operatorname{det}\left(\hat{\mathcal{R}}^{-}, \mathcal{R}^{+}, \hat{V}\right)\right|_{x_{1}=0,(\tilde{\xi}, \lambda)=(0,0)},
\end{aligned}
$$

where $\sigma= \pm 1$ depending on dimensions $n, r$ and $\operatorname{det}\left(\psi_{2}, \ldots, \psi_{n+1}\right) \neq 0$ assuming stability of the limiting shock $\hat{U}$ (else $\mathcal{D}$ would vanish to second instead of first order at the origin [Z3]). Normalizing

$$
\operatorname{sgn} \sigma \operatorname{det}\left(\psi_{2}, \ldots, \psi_{n+1}\right)=+1
$$

we obtain

$$
\lim _{X \rightarrow+\infty} \operatorname{sgn} \lim _{\lambda \rightarrow 0^{+} \text {real }} D_{X}(0, \lambda)=\hat{\delta}:=\operatorname{det}\left(\hat{R}^{-}, R_{+}, \hat{V}\right)
$$

as claimed.

Reviewing the computation in [Z3] of $\partial_{\lambda} \mathcal{D}(0,0)$, we find that this is the same normalization of $\psi_{j}$ columns leading to the assumed normalization (1.14), whence (1.15) is necessary for stability by the discussion above the statement of the theorem.

Finally, for a Lax 1-shock, all eigenvalues of $A_{1}^{-}$are positive; hence the $\mathcal{R}^{-}$block is empty in the computation above and the boundary conditions must be of inflow 
type. If also the boundary conditions are of Dirichlet type, then by (2.3), the first

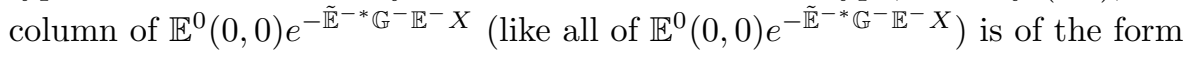

$$
\left.\left(\mathbb{E}^{0}(0,0) e^{-\tilde{\mathbb{E}}^{-*} \mathbb{G}^{-} \mathbb{E}^{-} X}\right)_{1}=\hat{\mathbb{E}}_{1}^{-}+\left(\hat{\mathbb{F}}^{-, \text {slow }} \alpha\right)_{1}=\left(\begin{array}{c}
0 \\
0 \\
*
\end{array}\right)\right),
$$

where $\hat{\mathbb{E}}_{1}^{-}$and $\hat{\mathbb{F}}_{1}^{-, \text {slow }}$, defined as the first column of $\hat{\mathbb{E}}^{-}:=T^{-}(-X) e^{-\mathbb{G}^{-} X} \mathbb{E}^{-}$and the slow part of $\hat{\mathbb{F}}^{-}:=T^{-}(-X) e^{-\mathbb{G}^{-} X} \mathbb{F}^{-}$, are asymptotically of the form (since $T^{-} e^{\mathbb{G}^{-} x_{1}} \mathbb{E}_{1}^{-}=\left(b_{11} \hat{U}^{\prime}\left(x_{1}\right), 0\right)$ and $\left.\lim _{z \rightarrow-\infty} T^{-}=I d\right)$

$$
\left|\hat{U}^{\prime}(-X)\right|\left(\begin{array}{c}
b_{11}^{-} S \\
0
\end{array}\right) \text { and }\left|\hat{U}^{\prime}(-X)\right|\left(\begin{array}{c}
b_{11}^{-}\left(-A_{1}^{-}\right)^{-1} \hat{\mathcal{R}}_{1}^{-} \\
\hat{\mathcal{R}}_{1}^{-}
\end{array}\right)
$$

as $X \rightarrow+\infty$, where $\hat{\mathcal{R}}_{1}^{-}$lies in the unstable subspace of $A_{1}^{-}$. Equating, we find that $\hat{\mathcal{R}}_{1}^{-}=\left(\begin{array}{l}0 \\ c\end{array}\right)$ and $b_{11}^{-} S=b_{11}^{-}\left(A_{1}^{-}\right)^{-1} \hat{\mathcal{R}}_{1}^{-}$. Recalling that $\left(A_{1}^{11}, A_{1}^{12}\right) \hat{U}^{\prime}=0$ by the linearized boundary-layer ODE, so that

$$
\left(\left(A_{1}^{-}\right)^{11},\left(A_{1}^{-}\right)^{12}\right) S=0
$$

we find by inspection that $\hat{\mathcal{R}}_{1}=A_{1}^{-} S$, or, in the notation of the general case,

$$
\hat{V}=A_{1}^{-} S,
$$

yielding the result.

Proof of Theorem 1.13. The proof follows immediately, by Lemma 3.2 and Theorem 1.11 .

Proof of Theorem 1.15. Set $\eta:=\left|\hat{U}^{\prime}(-X)\right|$, and consider $0 \leq \rho \leq C e^{-\theta X}$ for some fixed $C>0$ and $\theta>0$ sufficiently small, in particular, small enough that

$$
\left|\hat{U}^{\prime}(-X)\right| \ll C e^{-\theta X}
$$

setting

$$
(\tilde{\xi}, \lambda, \eta)=:\left(\rho \tilde{\xi}_{0}, \rho \tilde{\lambda}_{0}, \rho \eta_{0}\right)
$$

with $\left|\left(\tilde{\xi}_{0}, \tilde{\lambda}_{0}, \eta_{0}\right)\right|=1$. Note, as $X \rightarrow+\infty$, that the set of possible values of $\left(\tilde{\xi}_{0}, \tilde{\lambda}_{0}, \eta_{0}\right)$ expands to the full positive half-sphere $\Re \lambda_{0}, \eta_{0}>0$. Restrict now to a compact subset of the positive half-sphere, recalling (see Proposition 2.2) that the Evans functions $D_{X}$ and $\mathcal{D}$, and their component columns, are $C^{\infty}$ in $\rho, \tilde{\xi}_{0}, \tilde{\lambda}_{0}$.

Within the specified parameter regime, both slow and fast modes of (2.1) at $(\tilde{\xi}, \lambda)=\rho\left(\tilde{\xi}_{0}, \lambda_{0}\right)$ are well-approximated on $x_{1} \in[-X, 0]$ by their limiting values as $\rho \rightarrow 0$, described in Proposition 2.4 Mimicking the one-dimensional computations (4.5), (4.6), we may rewrite

$$
D_{X}\left(\rho \tilde{\xi}_{0}, \rho \lambda_{0}\right) / e^{\int_{0}^{-X} \operatorname{Trace} \mathbb{G}\left(\rho \tilde{\xi}_{0}, \rho \lambda_{0}, z\right) d z} e^{\operatorname{Trace}\left(\Pi_{\mathbb{E}} \mathbb{G}^{-}\right) X}
$$

as the sum of

$$
\rho \operatorname{det}\left(\begin{array}{ccccccccc}
* & \psi_{2} & \cdots & \psi_{k_{2}} & * & * & \psi_{k_{1}+1} & \cdots & \psi_{n+1} \\
\eta_{0} A_{1} S & 0 & \cdots & 0 & \hat{\mathcal{R}}^{-} & \mathcal{R}^{+} & 0 & \cdots & 0
\end{array}\right)+o\left(\rho \eta_{0}\right)
$$


and

$$
\begin{aligned}
& \rho \operatorname{det}\left(\begin{array}{ccccccccc}
* & \psi_{2} & \cdots & \psi_{k_{2}} & * & * & \psi_{k_{1}+1} & \cdots & \psi_{n+1} \\
\partial_{\rho} T^{-} \mathbb{E}_{1}^{-}-\partial_{\rho} T^{+} \mathbb{E}_{1}^{+} & 0 & \cdots & 0 & \hat{\mathcal{R}}^{-} & \mathcal{R}^{+} & 0 & \cdots & 0
\end{array}\right) \\
& \quad+o\left(\rho\left|\left(\tilde{\xi}_{0}, \lambda_{0}\right)\right|\right)
\end{aligned}
$$

both evaluated at $x_{1}=0, \rho=0$, and $\left(\tilde{\xi}_{0}, \lambda_{0}\right)$. We omit the details of this straightforward but tedious computation.

A standard computation [ZS, [Z3, GMWZ4] using the variational equations of (2.1) with respect to $\rho$ yields

$$
\partial_{\rho} T^{-} \mathbb{E}_{1}^{-}-\partial_{\rho} T^{+} \mathbb{E}_{1}^{+}=\lambda_{0}[U]+\sum_{j=2}^{d} i \tilde{\xi}_{0}^{j}\left[F_{j}(U)\right],
$$

whence, normalizing as usual so that

$$
\operatorname{sgn} \sigma \operatorname{det}\left(\psi_{2}, \ldots, \psi_{n+1}\right)=+1,
$$

we obtain by block determinant expansion,

$$
\begin{aligned}
\rho^{-1} D_{X}\left(\rho \tilde{\xi}_{0}, \rho \lambda_{0}\right) / e^{\int_{0}^{-X} \operatorname{Trace} \mathbb{G}\left(\rho \tilde{\xi}_{0}, \rho \lambda_{0}, z\right) d z} & e^{\operatorname{Trace}\left(\Pi_{\mathbb{E}}-\mathbb{G}^{-}\right) X} \\
& =\hat{\Delta}\left(\tilde{\xi}_{0}, \lambda_{0}, \eta_{0}\right)+o(1),
\end{aligned}
$$

where $\hat{\Delta}$ is defined as in (1.21) and $o(1)$ is $C^{1}$ with respect to $\tilde{\xi}_{0}, \lambda_{0}$, and $\eta_{0}$ for each fixed $X$ and $\rightarrow 0$ uniformly as $X \rightarrow 0$.

By an application of the Implicit Function Theorem, it follows that existence of a root $\left(\tilde{\xi}_{0}^{*}, \lambda_{0}^{*}, \eta_{0}^{*}\right)$ of $\hat{\Delta}$ on $\Re \lambda_{0}>0$ at which $\partial_{\lambda_{0}} \hat{\Delta} \neq 0$ implies existence of a nearby $\operatorname{root}\left(\tilde{\xi}_{0}^{*}, \lambda_{0}^{\dagger}, \tilde{\eta}_{0}^{*}\right), \Re \lambda_{0}^{\dagger}>0, \rho>0$, of

$$
\rho^{-1} D_{X}\left(\rho \tilde{\xi}_{0}, \rho \lambda_{0}\right) / e^{\int_{0}^{-X} \operatorname{Trace} \mathbb{G}\left(\rho \tilde{\xi}_{0}, \rho \lambda_{0}, z\right) d z} e^{\operatorname{Trace}\left(\Pi_{\mathbb{E}}-\mathbb{G}^{-}\right) X},
$$

hence of $D_{X}$, for $X$ sufficiently large, or instability of $\bar{U}^{X}$. Thus, nonvanishing of $\hat{\Delta}$ on the strictly positive half-sphere is necessary for stability as $X \rightarrow 0$.

Proof of Theorem 1.16. The estimate (4.8) in fact holds for all $\eta:=\left|\hat{U}^{\prime}(-X)\right|$ and $0 \leq \rho \leq C e^{-\theta X}$, with the $o(1)$ term uniformly decaying and uniformly $C^{0}$ as $X \rightarrow \infty$ (however, not uniformly $C^{1}$; see [GMWZ4, GMWZ5, GMWZ6]). It follows, therefore, that nonvanishing of $\hat{\Delta}$ on the (closed) nonnegative half-sphere, implying a lower bound on $|\hat{\Delta}|$, implies nonvanishing of $D_{X}$ on the parameter range $0 \leq \rho \leq$ $C e^{-\theta X}$, for $X$ sufficiently large.

If $C e^{-\theta X} \leq \rho \ll 1$, on the other hand, a much cruder estimate yields

$$
\begin{aligned}
\rho^{-1} D_{X}\left(\rho \tilde{\xi}_{0}, \rho \lambda_{0}\right) / e^{\int_{0}^{-X}} & \operatorname{Trace} \mathbb{G}\left(\rho \tilde{\xi}_{0}, \rho \lambda_{0}, z\right) d z \\
& =\Delta\left(\tilde{\xi}_{0}, \tilde{\lambda}_{0}\right)+o(1)+O\left(\left|\hat{U}^{\prime}(-X)\right| / C e^{-\theta X}\right) \\
& =\Delta\left(\tilde{\xi}_{0}, \tilde{\lambda}_{0}\right)+o(1),
\end{aligned}
$$

by (4.7), again with $o(1)$ uniformly decaying as $X \rightarrow+\infty$. This implies nonvanishing of $D_{X}$ on the parameter range $1 \gg \rho \geq C e^{-\theta X}$, for $X$ sufficiently large.

For $\rho$ bounded from below, on the other hand, we have by the basic convergence result of Theorem 1.8 that $D_{X}$ is nonvanishing if $\mathcal{D}$ and $D_{-}$are nonvanishing, i.e., if $\hat{U}$ and $U \equiv U_{-}$are stable. This completes the proof of the theorem. 


\section{Application to gas Dynamics}

We now apply our results to the fundamental example of compressible gas dynamics, restricting without loss of generality (by rotational invariance of the equations) to dimension $d=2$. Consider the compressible Navier-Stokes equations (5.1a)

$$
\begin{gathered}
(\rho u)_{t}+\left(\rho u^{2}+p\right)_{x}+(\rho u v)_{y}=(2 \mu+\eta) u_{x x}+\mu u_{y y}+(\mu+\eta) v_{x y} \\
(\rho v)_{t}+(\rho u v)_{x}+\left(\rho v^{2}+p\right)_{y}=\mu v_{x x}+(2 \mu+\eta) v_{y y}+(\mu+\eta) u_{y x} \\
(\rho E)_{t}+(\rho u E+u p)_{x}+(\rho v E+v p)_{y} \\
=\left(\kappa T_{x}+(2 \mu+\eta) u u_{x}+\mu v\left(v_{x}+u_{y}\right)+\eta u v_{y}\right)_{x} \\
+\left(\kappa T_{y}+(2 \mu+\eta) v v_{y}+\mu u\left(v_{x}+u_{y}\right)+\eta v u_{x}\right)_{y}
\end{gathered}
$$

on the half-plane $x \in \mathbb{R}^{+}, y \in \mathbb{R}$, where $\rho$ is density, $u$ and $v$ are velocities in the $x$ and $y$ directions, $p$ is pressure,

$$
E=e+\frac{u^{2}}{2}+\frac{v^{2}}{2}
$$

is total energy density, $e$ and $T$ are internal energy density and temperature, and the constants $\mu>|\eta| \geq 0$ and $\kappa>0$ are coefficients of first ("dynamic") and second viscosity and heat conductivity.

We assume ideal (" $\gamma$-law") gas equations of state

$$
p_{0}(\rho, T)=\Gamma \rho e, \quad e_{0}(\rho, T)=c_{v} T,
$$

where $c_{v}>0$ is the specific heat at constant volume, $\Gamma:=\gamma-1>0$, and $\gamma>1$ is the adiabatic index of the gas; equivalently,

$$
p(v, S)=a e^{S / c_{v}} \rho^{\gamma},
$$

where $S$ is thermodynamical entropy [Ba, $\mathrm{Sm}$. In the notation of (1.2), we have

$$
\tilde{U}=(\rho, \rho u, \rho v, \rho E)=:\left(\rho, m_{1}, m_{2}, \mathcal{E}\right)
$$

and

$$
F_{1}(\tilde{U})=\left(\rho u, \rho u^{2}+p, \rho v^{2}, \rho u E\right)=\left(m_{1}, m_{1}^{2} / \rho+p, m_{1} m_{2} / \rho, m_{1} \mathcal{E} / \rho\right) .
$$

Remark 5.1. In the thermodynamical rarified gas approximation,

$$
\gamma=\frac{2 n+3}{2 n+1}, \quad \nu / \mu=\frac{9 \gamma-5}{4} \quad \eta=-\frac{2}{3} \mu
$$

for $\nu:=\kappa / c_{v}$, where $n$ is the number of constituent atoms of gas molecules (here assumed to have "tree" structure) [Ba, with $\gamma=5 / 3$ and $\gamma=7 / 5$ for the main applications of monatomic and diatomic gas. In particular,

$$
1<\gamma<2 \text { and } \frac{\nu}{2 \mu+\eta}>1
$$

for common gases, a conclusion that is born out by experiment. See Appendices A and B of HLyZ1 for further discussion. 
5.1. Viscous shock profiles. From (5.1), setting time derivatives to zero, integrating in $x$, and rearranging, we obtain after a brief calculation the standing-shock ODE

$$
\begin{array}{r}
\hat{u}^{\prime}=(2 \mu+\eta)^{-1}\left(m\left(\hat{u}-u_{-}\right)+\Gamma\left(\hat{\rho} \hat{e}-\hat{\rho}_{-} e_{-}\right)\right), \\
\hat{e}^{\prime}=\nu^{-1}\left(m\left(\hat{e}-e_{-}\right)-\frac{m\left(\hat{u}-u_{-}\right)^{2}}{2}+\left(\hat{u}-u_{-}\right) \Gamma \hat{\rho}_{-} T_{-}\right),
\end{array}
$$

where $m:=\hat{\rho} \hat{u} \equiv$ constant and $\hat{v} \equiv$ constant.

Using various scale-invariances of system (5.1), we may take without loss of generality $m=\rho_{-}=u_{-}=1, v_{-}=v_{+}=0$, yielding

$$
\begin{array}{r}
\hat{u}^{\prime}=\frac{1}{2 \mu+\eta}\left((\hat{u}-1)+\Gamma\left(\frac{\hat{e}}{\hat{u}}-e_{-}\right)\right), \\
\hat{e}^{\prime}=\nu^{-1}\left(\left(\hat{e}-e_{-}\right)-\frac{(\hat{u}-1)^{2}}{2}+(\hat{u}-1) \Gamma e_{-}\right)
\end{array}
$$

with $\hat{v} \equiv 0$, with endstates

$$
e_{+}=\frac{u_{+} \alpha\left(u_{+}-1\right)}{\Gamma(\Gamma+2-\alpha)}, \quad e_{-}=\frac{\left(u_{+}-1\right)(\Gamma+2)}{\Gamma(\Gamma+2-\alpha)}, \quad \rho_{+}=1 / u_{+},
$$

$\alpha:=\frac{\Gamma+2-\Gamma u_{+}}{u_{+}-u_{*}}$, parametrized by the single quantity

$$
1 \geq u_{+}>u_{*}:=\frac{\Gamma}{\Gamma+2} \text {. }
$$

In the strong-shock limit, $u_{+} \rightarrow u_{*}, e_{-} \rightarrow 0$, with all other quantities remaining in physical range; for details of these computations, see HLyZ2, Sections 3-5.

Linearizing (5.10) about $\left(u_{-}, e_{-}\right)=\left(1, e_{-}\right)$, we obtain

$$
\left(\begin{array}{l}
u \\
e
\end{array}\right)^{\prime}=M_{-}\left(\begin{array}{l}
u \\
e
\end{array}\right), \quad M_{-}:=\left(\begin{array}{cc}
\frac{1}{2 \mu+\eta} & 0 \\
0 & \frac{1}{\nu}
\end{array}\right)\left(\begin{array}{cc}
1-\Gamma e_{-} & \Gamma \\
\Gamma e_{-} & 1
\end{array}\right),
$$

determining the asymptotic behavior of $(\hat{u}, \hat{e})(z)$ as $z \rightarrow-\infty$. One may check for all $1 \geq u_{+}>u_{*}$ that $M_{-}$has two positive distinct real eigenvalues $0<\omega_{-} \leq 1 / \nu \leq \omega_{+}$,

$$
\omega_{ \pm}=\frac{1}{\nu}+\frac{\left(\frac{1-\Gamma e_{-}}{2 \mu+\eta}-\frac{1}{\nu}\right) \pm \sqrt{\left(\frac{1-\Gamma e_{-}}{2 \mu+\eta}-\frac{1}{\nu}\right)^{2}+\frac{4 \Gamma e_{-}}{(2 \mu+\eta) \nu}}}{2},
$$

with associated eigenvectors $s_{j}=\left(-1,-\frac{\Gamma e_{-}}{\nu\left(\omega_{j}-1 / \nu\right)}\right)^{T}$, merging in the special limiting case $u_{+} \rightarrow u_{*} / e_{-} \rightarrow 0,2 \mu+\eta=\nu$ to a pair of real semisimple eigenvalues.

That is, for a Lax 1-shock, $U_{-}$is a repellor for the standing-wave ODE, and $U_{+}$a saddle, in agreement with the abstract conclusions of MaZ3] for extreme shocks of general systems and of [Gi] for shock profiles of gas dynamics with general equation of state. In particular, note that

$$
\operatorname{det} M_{-}=(\nu(2 \mu+\eta))^{-1}\left(1-\Gamma(1+\Gamma) e_{-}\right)>0,
$$

with $\left(1-\Gamma(1+\Gamma) e_{-}\right)$approaching 1 in the strong shock limit $u \rightarrow u_{*} / e_{-} \rightarrow 0$, and $1-\frac{2(\Gamma+1)}{(\Gamma+2)^{2}}>\frac{\Gamma^{2}+2}{(\Gamma+2)^{2}}$ in the weak shock limit $u_{+} \rightarrow 11$ By reality and simplicity of the eigenvalues $\omega_{j}$, we have that the limits

$$
s:=\lim _{z \rightarrow-\infty}\left(\left(\hat{u}^{\prime}, \hat{e}^{\prime}\right) /\left|\left(\hat{u}^{\prime}, \hat{e}^{\prime}\right)\right|\right)
$$

\footnotetext{
${ }^{1}$ This repairs an error of [SZ, in which $U_{-}$was mistakenly computed to be a saddle, leading to an incorrect value of $S$.
} 
and

$$
S:=\lim _{z \rightarrow-\infty}\left(\hat{U}^{\prime} /\left|\hat{U}^{\prime}\right|\right)=\left.\frac{\partial U}{\partial(u, e)}\right|_{U_{-}} s=\left(\begin{array}{cc}
-1 & 0 \\
0 & 0 \\
0 & 0 \\
1 / 2-e_{-} & 1
\end{array}\right) s
$$

exist, with $s$ generically lying parallel to the slow mode $s_{-}$, or

$$
\begin{aligned}
S & =\left(\begin{array}{cc}
-1 & 0 \\
0 & 0 \\
0 & 0 \\
1 / 2-e_{-} & 1
\end{array}\right)\left(\begin{array}{c}
-1 \\
-\frac{\Gamma e_{-}}{\nu\left(\omega_{-}-1 / \nu\right)}
\end{array}\right) \\
& =\left(\begin{array}{c}
1 \\
0 \\
0 \\
e_{-} \frac{\Gamma-\nu\left(\omega_{-}-1 / \nu\right)}{\nu\left(\omega_{-}-1 / \nu\right)}-\frac{1}{2}
\end{array}\right) .
\end{aligned}
$$

Finally, from (5.5) -(5.6), we obtain, after a brief calculation,

$$
\begin{aligned}
A_{1}^{-}:=\partial\left(F_{1} / \partial \tilde{U}\right)\left(U_{-}\right) & =\left(\begin{array}{cccc}
0 & 1 & 0 & 0 \\
p_{\rho}-1+p_{e} / 2 & 2-p_{e} & 0 & p_{e} \\
0 & 0 & 1 & 0 \\
-1 / 2 & 1 / 2 & 0 & 1
\end{array}\right) \\
& =\left(\begin{array}{cccc}
\Gamma e_{-}-1+\Gamma / 2 & 2-\Gamma / 2 & 0 & \Gamma \\
0 & 0 & 1 & 0 \\
-1 / 2 & 1 / 2 & 0 & 1
\end{array}\right),
\end{aligned}
$$

from which we compute

$$
\begin{gathered}
A_{1}^{-} S=\left(\begin{array}{c}
0 \\
\Gamma e_{-}-1+\Gamma / 2+\Gamma e_{-} \frac{\Gamma-\nu\left(\omega_{-}-1 / \nu\right)}{\nu\left(\omega_{-}-\Gamma / \nu\right)} \\
0 \\
e_{-} \frac{\Gamma-\nu\left(\omega_{-}-1 / \nu\right)}{\nu\left(\omega_{-}-1 / \nu\right)}-1
\end{array}\right), \\
{[U]=\left(\frac{1-u_{+}}{u_{+}}, 0,0, \frac{1-u_{+}}{2}\right)^{T} .}
\end{gathered}
$$

5.1.1. The strong shock limit. For $\frac{\nu}{2 \mu+\eta}<1$, (5.16) converges to $S=(1,0,0,-1 / 2)^{T}$ in the strong shock limit $e_{-} \rightarrow 0$. For $\frac{\nu}{2 \mu+\eta} \geq 1$, however, (5.16) becomes singular in the limit as $e_{-} \rightarrow 0$, for which also $\omega_{-} \rightarrow 1 / \nu$. To evaluate this limit, it is easier to return to (5.13) and compute directly with $e_{-}=0$, to obtain $s \rightarrow(-1,1-\phi)^{T}$, yielding the general formula

$$
S \rightarrow(1,0,0,1 / 2-\min \{1, \phi\})^{T} \text { as } u_{+} \rightarrow u_{-}, \quad \phi:=\frac{2 \mu+\eta}{\nu} .
$$

Noting that

$$
A_{1}-\rightarrow\left(\begin{array}{cccc}
0 & 1 & 0 & 0 \\
-1+\Gamma / 2 & 2-\Gamma / 2 & 0 & \Gamma \\
0 & 0 & 1 & 0 \\
-1 / 2 & 1 / 2 & 0 & 1
\end{array}\right)
$$


we thus have

$$
A_{1} S-\rightarrow\left(\begin{array}{c}
0 \\
\Gamma \max \{0,1-\phi\}-1 \\
0 \\
-\min \{1, \phi\}
\end{array}\right)
$$

and

$$
[U] \rightarrow\left(\frac{2}{\Gamma}, 0,0, \frac{1}{\Gamma+2}\right)^{T}
$$

completing our asymptotic analysis.

5.2. One-dimensional stability. Following the treatment in [Se1, SZ, we note for Lax 1-shocks that

$$
\operatorname{det}\left(\mathcal{R}_{+}, f\right)=\ell_{+} \cdot f
$$

for any vector $f \in \mathbb{C}^{n}$, where $\ell_{+}$is the unique stable left eigenvector of $\mathcal{A}_{+}$and $\cdot$ denotes complex inner product. In one dimension, $\ell_{+}$is just the stable left eigenvector of $A_{+}$, which may be computed to be

$$
\begin{aligned}
\ell_{+} & =\left(p_{\rho}+c u+\frac{p_{e}\left(u^{2} / 2-e\right)}{\rho},-\frac{p_{e} u}{\rho}-c, 0, \frac{p_{e}}{\rho}\right)^{T}\left(U_{+}\right) \\
& =\left(c_{+} u_{+}+\Gamma u_{+}^{2} / 2,-\Gamma u_{+}-c_{+}, 0, \Gamma\right)^{T},
\end{aligned}
$$

where

$$
c:=\sqrt{p p_{e} / \rho^{2}+p_{\rho}}=\sqrt{\Gamma(\Gamma+1) e}
$$

denotes sound speed. This computation is most easily accomplished by working in the more convenient nonconservative coordinates $(\rho, u, v, e)$, which are related to conservative variables $\left(\rho, \rho u, \rho v, \rho\left(e+u^{2} / 2+v^{2} / 2\right)\right)$ by a readily computed lower triangular change of coordinates; see [Se1] or Appendix $\mathrm{A}$.

Combining all facts, we have

$$
\begin{aligned}
\hat{\delta}= & \ell_{+} \cdot A_{-} S \\
= & \left(-\Gamma u_{+}-c_{+}\right)\left(\Gamma e_{-}-1+\Gamma / 2+\Gamma e_{-} \frac{\Gamma-\nu\left(\omega_{-}-1 / \nu\right)}{\nu\left(\omega_{-}-\Gamma / \nu\right)}\right) \\
& +\Gamma\left(e_{-} \frac{\Gamma-\nu\left(\omega_{-}-1 / \nu\right)}{\nu\left(\omega_{-}-1 / \nu\right)}-1\right) .
\end{aligned}
$$

It is readily verified on the other hand that

$$
\delta>0
$$

see Section 5.2.1 just below. The one-dimensional stability condition (1.17) thus reduces in this case to

$$
\operatorname{sgn} \hat{\delta}>0,
$$

a condition that can be readily checked numerically using (5.27). 
5.2.1. The strong shock limit. In the strong shock limit $u_{+} \rightarrow u_{*}$, we have $e_{-} \rightarrow 0$, $\alpha \rightarrow+\infty$, and

$$
e_{+} \rightarrow u_{*}\left(1-u_{*}\right) / \Gamma=2 /(\Gamma+2)^{2},
$$

so that $c_{+} \rightarrow \sqrt{2 \Gamma(\Gamma+1)} /(\Gamma+2)$ and

$$
\ell_{+} \rightarrow\left(\frac{\Gamma \sqrt{2 \Gamma(\Gamma+1)}}{(\Gamma+2)^{2}}+\frac{\Gamma^{3}}{2(\Gamma+2)^{2}},-\frac{\Gamma^{2}}{\Gamma+2}-\frac{\Gamma \sqrt{2 \Gamma(\Gamma+1)}}{\Gamma+2}, 0, \Gamma\right)^{T} ;
$$

hence, by (5.22),

$$
\begin{aligned}
\hat{\delta} & =\ell_{+} \cdot A_{-} S \\
& \rightarrow\left(-\frac{\Gamma^{2}}{\Gamma+2}-\frac{\Gamma \sqrt{2 \Gamma(\Gamma+1)}}{\Gamma+2}\right)(\Gamma \max \{0,1-\phi\}-1)-\Gamma \min \{1, \phi\} .
\end{aligned}
$$

Meanwhile,

$$
\delta=\ell_{+} \cdot[U] \rightarrow \frac{2 \sqrt{2 \Gamma(\Gamma+1)}}{(\Gamma+2)^{2}}+\frac{\Gamma}{(\Gamma+2)^{2}}+\frac{\Gamma}{\Gamma+2}>0,
$$

from which we may conclude by homotopy/nonvanishing of $\delta$ that $\delta>0$ for all $1 \geq u_{+} \geq u_{*}$, verifying (5.28) (5.29).

The case $\phi \geq 1$. For $\phi \geq 1$, (5.29) becomes

$$
\left(\frac{\Gamma \sqrt{2 \Gamma(\Gamma+1)}}{\Gamma+2}+\frac{\Gamma^{2}}{\Gamma+2}\right)-\Gamma>0
$$

or

$$
\sqrt{2 \Gamma(\Gamma+1)}>2
$$

which evidently fails for $\Gamma$ in the kinetic range $0 \leq \Gamma \leq 1$ (indeed, for all $\Gamma$ outside $(1,2))$. Thus, we may conclude instability in the strong shock limit in this range.

The case $\phi \leq 1$. For $\phi \leq 1,(5.29)$ becomes

$$
\left(-\frac{\Gamma^{2}}{\Gamma+2}-\frac{\Gamma \sqrt{2 \Gamma(\Gamma+1)}}{\Gamma+2}\right)(\Gamma(1-\phi)-1)-\Gamma \phi>0
$$

or

$$
(\sqrt{2 \Gamma(\Gamma+1)}+\Gamma)(1-\Gamma(1-\phi))-(\Gamma+2) \phi>0 .
$$

Defining $\sigma:=\sqrt{2 \Gamma(\Gamma+1)}+\Gamma$, we may rewrite this as

$$
\sigma(1-\Gamma)>(\Gamma-\Gamma \sigma+2) \phi,
$$

or, assuming $\Gamma(1-\sigma)+2>0$, as holds for example on the kinetic range $0<\Gamma<1$, or $1<\gamma<2$ (on which $\sigma<2+\Gamma$, so $2+\Gamma>\Gamma \sigma$ ), as

$$
\phi<\frac{\sigma(1-\Gamma)}{\Gamma(1-\sigma)+2},
$$

which is satisfied for $\phi$ small enough, but for $\phi=1$, hence for $\phi \leq 1$ large enough, is not satisfied, by the analysis of the case $\phi=1$ above. 
Common gases and the kinetic approximation. Recall that for common gases, $\phi$ is less than one. For gases obeying the kinetic approximation (5.7)-(5.8),

$$
\phi=\frac{16}{27 \Gamma+12},
$$

so that $\phi<1$ for $\Gamma \geq 4 / 27 \approx .148$, in particular for $n$-atomic gases with $n \leq 5$. Thus, it is the case $\phi \leq 1$ that is relevant to typical applications. Substituting (5.32) into (5.31) and noting that $\sqrt{2 \Gamma(\Gamma+1)} \leq \Gamma+1$ for $0<\Gamma<1$ yields the necessary condition

$$
16(\Gamma+2)<(2 \Gamma+1)(1+15 \Gamma),
$$

or $0<(\Gamma-1)(30 \Gamma+31)$, which is violated for the entire kinetic range $0<\Gamma<1$.

Conclusions. By Remarks 1.12 2 and 1.18,2, boundary layers are both one- and multi-dimensionally stable in the standing shock limit for limiting shocks of sufficiently small amplitude, i.e., $1-u_{+}$sufficiently small. By the calculations above, however, for typical gas laws, they are not even one-dimensionally stable in the strong shock limit for limiting shocks of sufficiently large amplitude, i.e., $u_{+}-u_{*}$ sufficiently small, even though the corresponding shock is perfectly stable HLyZ1, HLyZ2.

Thus, we have the striking conclusion that for (all!) typically physically occurring gases under inflow Dirichlet boundary conditions, there is a transition from stability to instability of boundary layers in the standing shock limit as the amplitude of the limiting shock increases from zero to its maximum value.

5.3. Multi-dimensional stability. The computation of $\ell_{+}(\tilde{\xi}, \lambda)$ in multi-dimensions may be found, for example, in Appendix $\mathrm{C}$ in [Z3] 2 , where it is computed as

$$
\ell_{+}(\tilde{\xi}, \lambda)=\left(\theta-\frac{i c \beta u}{\sqrt{\tilde{\xi}^{2}-\beta^{2}}}+\frac{\eta u^{2}}{\beta}, \frac{i c \beta}{\sqrt{\tilde{\xi}^{2}-\beta^{2}}}-\eta u, \frac{c \tilde{\xi}}{\sqrt{\tilde{\xi}^{2}-\beta^{2}}}, \eta\right)_{+},
$$

where $\theta:=p_{\rho}-\frac{p_{e} e}{\rho}=2 \Gamma e, \eta:=\frac{p_{e}}{\rho}=\Gamma$, and $c$ is sound speed (5.26), or

$$
\ell_{+}(\tilde{\xi}, \lambda)=\left(2 \Gamma e_{+}-\frac{i c_{+} \beta_{+} u_{+}}{\sqrt{\tilde{\xi}^{2}-\beta_{+}^{2}}}+\frac{\Gamma u_{+}^{2}}{\beta_{+}}, \frac{i c_{+} \beta_{+}}{\sqrt{\tilde{\xi}^{2}-\beta_{+}^{2}}}-\Gamma u_{+}, \frac{c_{+} \tilde{\xi}}{\sqrt{\tilde{\xi}^{2}-\beta_{+}^{2}}}, \Gamma\right)
$$

where

$$
\beta:=\frac{-u \lambda-\sqrt{\lambda^{2}+\tilde{\xi}^{2}\left(c^{2}-u^{2}\right)}}{c^{2}-u^{2}} .
$$

Together with our computation of $A_{1}^{-} S$ in (5.18), this determines $\ell_{+} \cdot A_{1}^{1} S$. Meanwhile, $\Delta:=\ell_{+} \cdot\left(\lambda[U]+i \tilde{\xi}\left[F_{2}(U)\right]\right)$ is computed for the same choice of $\ell_{+}$in Appendix C, Z3 (equation displayed below C.36), thus determining $\hat{\eta}(\tilde{\xi}, \lambda)=$ $\ell_{+} \cdot A_{1}^{1} S / \Delta(\tilde{\xi}, \lambda)$.

With Proposition 1.19 this gives a straightforward means of numerical determination of multi-dimensional instability, by plotting the image of $\hat{\eta}(1, i \tau)$ as $\tau$ ranges over the real axis and checking whether or not this curve strikes the nonnegative real axis; however, we shall not carry this out here.

\footnotetext{
${ }^{2}$ Contributed by K. Jenssen and G. Lyng
} 
The numerical determination of one- and multi-dimensional stability transitions for ideal and other gas laws would be interesting problems for further investigation. A further very interesting open problem is to determine analytically the stability transitions as was done for the inviscid shock problem (involving only $\Delta$ ) in [Er,, $\mathrm{M}]$; see Appendix $\mathrm{C}$ in [Z3.

\section{Appendix A. Computation of $\ell_{+}$in One Dimension}

In this appendix, we carry out for completeness the computation of $\ell_{+}$for the one-dimensional Navier-Stokes equations, verifying (5.25). In variables $(\rho, u, v, e)$, the quasilinear hyperbolic part of the equations becomes

$$
\begin{aligned}
& \rho_{t}+q \cdot \nabla \rho+\rho \operatorname{div} q=0 \\
& q_{t}+q \cdot \nabla q+\rho^{-1} p_{\rho} \nabla \rho+\rho^{-1} p_{e} \nabla e=0, \\
& e_{t}+q \cdot \nabla e+\rho^{-1} p \operatorname{div} u=0
\end{aligned}
$$

where $q=(u, v)$ denotes velocity, or, in one dimension,

$$
V_{t}+(u I d+M) V_{x_{1}}=0
$$

where $V=(\rho, u, v, e)$ and

$$
M:=\left(\begin{array}{cccc}
0 & \rho & 0 & 0 \\
\rho^{-1} p_{\rho} & 0 & 0 & \rho^{-1} p_{e} \\
0 & 0 & 0 & 0 \\
0 & \rho^{-1} p & 0 & 0
\end{array}\right),
$$

from which we may conclude that $A_{1}=S(u I d+M) S^{-1}$ for

$$
\begin{gathered}
S:=\frac{\partial\left(\rho, \rho u, \rho v, \rho\left(e+u^{2} / 2+v^{2} / 2\right)\right)}{\partial(\rho, u, v, e)}=\left(\begin{array}{ccccc}
1 & 0 & 0 & 0 \\
u & \rho & 0 & 0 \\
v & 0 & \rho & 0 \\
e+u^{2} / 2+v^{2} / 2 & \rho u & \rho v & \rho
\end{array}\right), \\
S^{-1}:=\left(\begin{array}{cccc}
1 & 0 & 0 & 0 \\
\frac{-u}{\rho} & \frac{1}{\rho} & 0 & 0 \\
\frac{-v}{\rho} & 0 & \frac{1}{\rho} & 0 \\
\frac{-e+u^{2} / 2+v^{2} / 2}{\rho} & \frac{-u}{\rho} & \frac{-v}{\rho} & \frac{1}{\rho}
\end{array}\right),
\end{gathered}
$$

and thus $\ell_{+}^{*}=\tilde{\ell}_{+}^{*} S^{-1}$ for $\tilde{\ell}_{+}$defined as the left eigenvector of $M$ associated with the eigenvalue of smallest real part, $*$ denoting adjoint, or congugate transpose, all quantities to be evaluated at $(\rho, u, v, e)=\left(1 / u_{+}, u_{+}, 0, e_{+}\right)$.

By inspection, $\tilde{\ell}_{+}^{*}=\left(p_{\rho},-\rho c, 0, p_{e}\right)$ for $v=0$, where the sound speed $c$ is defined as in (5.26), whence

$$
\ell_{+}^{*}=\tilde{\ell}_{+}^{*} S^{-1}=\left(p_{\rho}+c u+\frac{p_{e}\left(u^{2} / 2-e\right)}{\rho},-\frac{p_{e} u}{\rho}-c, 0, \frac{p_{e}}{\rho}\right)\left(U_{+}\right),
$$

as claimed. 


\section{REFERENCES}

[AGJ] Alexander, J., Gardner, R. and Jones, C.K.R.T., A topological invariant arising in the analysis of traveling waves. J. Reine Angew. Math. 410 (1990), 167-212. MR1068805 (92d:58028)

[Ba] Batchelor, G. K., An introduction to fluid dynamics. Cambridge Mathematical Library. Cambridge University Press, Cambridge, paperback edition, 1999. MR1744638 (2000j:76001)

[CHNZ] Costanzino, N., Humpherys, J., Nguyen, T., and Zumbrun, K., Spectral stability of noncharacteristic boundary layers of isentropic Navier-Stokes equations, Arch. Ration. Mech. Anal. 192 (2009), no. 3, 537-587.

[Er] Erpenbeck, J. J., Stability of step shocks, Phys. Fluids 5 (1962) no. 10, 1181-1187. MR0155515 (27:5449)

[FS] Freistühler, H. and Szmolyan. P., Spectral stability of small-amplitude viscous shock waves in several dimensions, To appear, Archive for Rat. Mech. Anal.

[GG] Grenier, E. and Guès, O., Boundary layers for viscous perturbations of noncharacteristic quasilinear hyperbolic problems, J. Differential Eqns. 143 (1998), 110-146. MR:1604888 (98j:35026)

[GMWZ4] Guès, O., Métivier, G., Williams, M., and Zumbrun, K., Paper 4, Navier-Stokes regularization of multidimensional Euler shocks, Ann. Sci. École Norm. Sup. (4) 39 (2006), no. 1, 75-175. MR 2224659 (2006m:35242)

[GMWZ5] Guès, O., Métivier, G., Williams, M., and Zumbrun, K., Existence and stability of noncharacteristic boundary layers for the compressible Navier-Stokes and MHD equations, to appear, Arch. Rational. Mech. Anal.

[GMWZ6] Guès, O., Métivier, G., Williams, M., and Zumbrun, K., Viscous boundary value problems for symmetric systems with variable multiplicities, J. Diff. Eq. 244 (2008), 309387. MR2376200 (2009c:35280)

[GR] Grenier, E. and Rousset, F., Stability of one dimensional boundary layers by using Green's functions, Comm. Pure Appl. Math. 54 (2001), 1343-1385. MR1846801 (2003a:35126)

[GZ] Gardner, R. and Zumbrun, K., The gap lemma and geometric criteria instability of viscous shock profiles, Comm. Pure Appl. Math. 51, 1998, 797-855. MR1617251 (99c:35152)

[Gi] Gilbarg, D., The existence and limit behavior of the one-dimensional shock layer. Amer. J. Math. 73:256-274, 1951. MR0044315(13:401e)

[HLyZ1] Humpherys, J., Lyng, G., and Zumbrun, K., Spectral stability of ideal-gas shock layers, Arch. Ration. Mech. Anal. 194 (2009), no. 3, 1029-1079. MR2563632

[HLyZ2] Humpherys, J., Lyng, G., and Zumbrun, K., Multidimensional spectral stability of large-amplitude Navier-Stokes shocks, in preparation.

[HuZ] Humpherys, J. and Zumbrun, K., Spectral stability of small amplitude shock profiles for dissipative symmetric hyperbolic-parabolic systems. Z. Angew. Math. Phys. 53 (2002) 20-34. MR:1889177 (2003b:35133)

[Kat] Kato, T., Reprint of the 1980 edition, Perturbation theory for linear operators. Springer-Verlag, Berlin, Heidelberg, (1995). MR1335452 (96a:47025)

[KK] Kagei, Y. and Kawashima, S., Stability of planar stationary solutions to the compressible Navier-Stokes equations in the half space, Comm. Math. Phys. 266 (2006), 401-430. MR.2238883 (2007g:35186)

[KNZ] Kawashima, S., Nishibata, S. and Zhu, P., Asymptotic stability of the stationary solution to the compressible Navier-Stokes equations in the half space, Comm. Math. Phys. 240 (2003), no. 3, 483-500. MR2005853 (2004g:76103)

[M] Majda, A., The stability of multi-dimensional shock fronts - a new problem for linear hyperbolic equations. Mem. Amer. Math. Soc. 275 (1983). MR683422 (84e:35100)

[MaZ3] Mascia, C. and Zumbrun, K., Pointwise Green function bounds for shock profiles of systems with real viscosity, Arch. Ration. Mech. Anal. 169 (2003), no. 3, 177-263. MR.2004135 (2004h:35137)

[MN] Matsumura, A. and Nishihara, K., Large-time behaviors of solutions to an inflow problem in the half space for a one-dimensional system of compressible viscous gas, Comm. Math. Phys., 222 (2001), no. 3, 449-474. MR.1888084 (2002m:76083) 
[MZ1] Métivier, G. and Zumbrun, K., Viscous Boundary Layers for Noncharacteristic Nonlinear Hyperbolic Problems, Memoirs AMS, 826 (2005).

[MZ2] Métivier, G. and Zumbrun, K., Hyperbolic Boundary Value Problems for Symmetric Systems with Variable Multiplicities, J. Diff. Equ., 211 (2005) 61-134. MR2121110 $(2005 \mathrm{j}: 35145)$

[N1] Nguyen, T., Stability of multidimensional viscous shocks for symmetric systems with variable multiplicities, Duke Math. J. 150 (2009), no. 3, 577-614. MR2582105

[N2] Nguyen, T., Long-time stability of viscous MHD boundary layers, preprint (2008).

[NZ1] Nguyen, T. and Zumbrun, K., Long-time stability of large-amplitude noncharacteristic boundary layers for hyperbolic parabolic systems, J. Math. Pures Appl. (9) 92 (2009), no. 6, 547-598. MR2565843

[NZ2] Nguyen, T. and Zumbrun, K., Long-time stability of multi-dimensional noncharacteristic viscous boundary layers, to appear, Comm. Math. Phys.

[PZ] Plaza, R. and Zumbrun, K., An Evans function approach to spectral stability of smallamplitude shock profiles, Discrete Contin. Dyn. Syst. 10 (2004) 885-924. MR2073940 (2005d:35169)

[R3] Rousset, F., Stability of small amplitude boundary layers for mixed hyperbolicparabolic systems, Trans. Amer. Math. Soc. 355 (2003), no. 7, 2991-3008. MR1975409 (2004h:35094)

[Se1] Serre, D., La transition vers l'instabilité pour les ondes de chocs multi-dimensionnelles, Trans. Amer. Math. Soc. 353 (2001) 5071-5093. MR.1852095 (2002m:35149)

[Se2] Serre, D., Sur la stabilité des couches limites de viscosité, (French. English, French summary) [Stability of viscosity boundary layers] Ann. Inst. Fourier (Grenoble) 51 (2001), no. 1, 109-130. MR1821071 (2002a:35141)

[SZ] Serre, D. and Zumbrun, K., Boundary layer stability in real vanishing-viscosity limit, Comm. Math. Phys. 221 (2001), no. 2, 267-292. MR.1845324 (2003m:35032)

[Sm] Smoller, J., Shock waves and reaction-diffusion equations. Springer-Verlag, New York, second edition, 1994. MR 1301779 (95g:35002)

[YZ] Yarahmadian, S. and Zumbrun, K., Pointwise Green function bounds and long-time stability of large-amplitude noncharacteristic boundary layers, SIAM J. Math. Anal. 40 (2009), 2328-2350. MR 2481297 (2009k:35188)

[Z1] Zumbrun, K., Multidimensional stability of planar viscous shock waves, Advances in the theory of shock waves, 304-516. Progress in Nonlinear PDE, 47, Birkhäuser, Boston, 2001. MR1842778 (2002k:35200)

[Z3] Zumbrun, K., Stability of large-amplitude shock waves of compressible Navier-Stokes equations. Handbook of Fluid Mechanics III, S.Friedlander, D.Serre, eds., Elsevier North Holland (2004). MR2099037 (2006f:35229)

[ZH] Zumbrun, K. and Howard, P., Pointwise semigroup methods and stability of viscous shock waves, Indiana Univ. Math. J. 47 (1998), 741-871. MR1665788 (99m:35157)

[ZS] Zumbrun, K. and Serre, D. Viscous and inviscid stability of multidimensional planar shock fronts, Indiana Univ. Math. J. 48. (1999), 937-992. MR1736972(2001h:35122)

Department of Mathematics, Indiana University, Bloomington, Indiana 47405

E-mail address: kzumbrun@indiana.edu 